\title{
Radical Cation $\pi$-Dimers of Conjugated Oligomers as Molecular Wires: An Analysis Based on Nitronyl Nitroxide Spin Labels
}

Tohru Nishinaga, Yuki Kanzaki, Daisuke Shiomi, Kenji Matsuda, Shuichi Suzuki, Keiji Okada

\begin{tabular}{|c|l|}
\hline Citation & Chemistry - A European Journal, 24(45); 11717-11728 \\
\hline Issue Date & 2018-08-09 \\
\hline Type & Journal Article \\
\hline Textversion & Author \\
\hline Rights & $\begin{array}{l}\text { This is the peer reviewed version of the following article: Chemistry - A } \\
\text { European Journal, Vol.24, Issu.45, p.11717-11728, which has been published in } \\
\text { final form at https://doi.org/10.1002/chem.201801712 . This article may be used } \\
\text { for non-commercial purposes in accordance with Wiley Terms and Conditions } \\
\text { for Use of Self-Archived Versions. }\end{array}$ \\
\hline DOI & \begin{tabular}{l}
$10.1002 / c h e m .201801712$ \\
\hline
\end{tabular}
\end{tabular}

\author{
Self-Archiving by Author(s) \\ Placed on: Osaka City University
}

NISHINAGA T., KANZAKI Y., SHIOMI D., SUZUKI S., OKADA K., MATSUDA K., \& SUZUKI S. (2018). Radical Cation $\Pi$-Dimers of Conjugated Oligomers as Molecular Wires: An Analysis Based on Nitronyl Nitroxide Spin Labels. Chemistry - A European Journal. 24, 11717-11728. 


\title{
Radical Cation $\pi$-Dimers of Conjugated Oligomers as Molecular Wires: an Analysis based on Nitronyl Nitroxide Spin Labels
}

\author{
Tohru Nishinaga, ${ }^{*[a]}$ Yuki Kanzaki, ${ }^{[b]}$ Daisuke Shiomi, ${ }^{*[b]}$ Kenji Matsuda, ${ }^{[c]}$ Shuichi Suzuki, ${ }^{[b, d]}$ and Keiji \\ Okada $^{[b]}$
}

\begin{abstract}
Nitronyl nitroxide (NN)-substituted conjugated oligomers, which were expected to self-associate in biradical cation states, were designed to analyze the capability of $\pi$-dimers as molecular wires. The oligomer moieties were composed of dithienyl- $N$-methylpyrrole with methoxy substituents at the inner $\beta$-position of thiophene rings (DTP. NN) and its propylenedioxythiophene (ProDOT) inserted derivative (DTP-P-NN'), or two ethylenedioxythiophene (EDOT) and two ProDOT units $\left(\mathbf{E}_{2} \mathbf{P}_{2}-\mathbf{N N}\right)^{*}$ ). Among them, chemical one-electron oxidation gave biradical cations (DTP-P) ${ }^{*+}-\mathbf{N N}^{*}$ and $\left(\mathrm{E}_{2} \mathbf{P}_{2}\right)^{*+}-\mathbf{N N}^{\cdot}$ that formed $\pi$-dimers (DTP-P-NN $)_{2}{ }^{2+}$ and $\left(\mathrm{E}_{2} \mathrm{P}_{2}-\mathrm{NN}\right)_{2}{ }^{2+}$ in dichloromethane at low temperatures. ESR studies of (DTP-P-NN $)_{2}{ }^{2+}$ and $\left(E_{2} P_{2}-N N\right)_{2}{ }^{2+}$ showed the presence of a relatively strong exchange interaction between two $\mathrm{NN}$ radicals through the radical cation $\pi$-dimer moieties. DFT calculations supported these experimental results and predicted that exchange interactions between two NN radicals were comparable or stronger than those through covalently linked quaterthiophene. Thus, the conjugated oligomer radical cation $\pi$-dimers acted as efficient molecular wires for electronic communication.
\end{abstract}

\section{Introduction}

In the last two decades, molecular wires ${ }^{[1-3]}$ have attracted enormous interest as fundamental components for molecular electronics. ${ }^{[4,5]}$ Molecular wires for molecular electronic devices mediate electron or charge transport, and typically consist of linear $\pi$-conjugated systems. ${ }^{[6-12]}$ In addition to covalently linked $\pi$-conjugated bridges, the effects of hydrogen bonds, ${ }^{[13-15]}$ metalmetal interactions, ${ }^{[16-18]}$ and coordination bonds ${ }^{[16,19,20]}$ on molecular wire properties have also been investigated. These weak bonds are beneficial for constructing self-assembled

\section{[a] Prof. Dr. T. Nishinaga}

Department of Chemistry, Graduate School of Science, Tokyo Metropolitan University, Hachioji, Tokyo 192-0397, Japan. E-mail: nishinaga-tohru@tmu.ac.jp

[b] Dr. Y. Kanzaki, Prof. Dr. D. Shiomi, Prof. Dr. S. Suzuki, Prof. Dr. K Okada

Department of Chemistry, Graduate School of Science, Osaka City University, Sumiyoshi-ku, Osaka 558-8585, Japan.

E-mail: shiomi@sci.osaka-cu.ac.jp

[c] Prof. Dr. K. Matsuda

Department of Synthetic Chemistry and Biological Chemistry, Graduate School of Engineering, Kyoto University, Katsura, Nishikyo-ku, Kyoto, 615-8510, Japan.

[d] Present Address: Division of Chemistry, Graduate School of Engineering Science, Osaka University.

Supporting information for this article is given via a link at the end of the document. systems and as conventional intermolecular interactions in supramolecular chemistry. Recently, as an emerging weak interaction, the use of long-bonded dimers of stable radical ions, ${ }^{[21,22]}$ the so-called $\pi$-dimers, has been expanded to control molecular motion ${ }^{[23]}$ and to construct supramolecular architectures. ${ }^{[24]}$ In addition to these applications, $\pi$-dimer-based conductive supramolecular wires present another interesting challenge. For this purpose, radical cation $\pi$-dimers of conjugated oligomers are the best candidates for supramolecular synthons.

Initially, $\pi$-dimers of conjugated oligomer radical cations were studied as a model for conductive polymers in the positively doped (p-doped) state. ${ }^{[25,26]}$ From the diamagnetic character of the terthiophene radical cation $\pi$-dimer, it was proposed that the $\pi$-dimeric interchain interaction can act as an alternative charge carrier to bipolaron (an intrachain closed-shell dication) in macroscopic conduction. ${ }^{[27]}$ Since then, various oligothiophenebased $\pi$-dimers have been prepared to understand the electronic structure of p-doped polythiophenes. ${ }^{[28-38]}$ Nevertheless, microscopic conductive properties of long-bonded $\pi$-dimers as molecular wire have not been verified owing to the absence of a suitable model system. Knowledge of these properties would be indispensable for the application of $\pi$-dimer-based conductive supramolecular wires.

The magnetic properties of the $\pi$-dimers are also of interest, and may indicate the potential spintronics applications based on conjugated oligomers. Superconducting quantum interference devices (SQUIDs) are powerful tools for studying the magnetic properties of materials. A pure sample is desired for the analysis of magnetic properties using a SQUID. However, crystalline samples of oligothiophene radical cations with a $\pi$-dimer structure are rare. ${ }^{[39-41]}$ Therefore, the lack of pure samples with structural information has hampered the detailed investigation of the magnetic properties of the $\pi$-dimers.

Nitronyl nitroxide $(\mathrm{NN})$ is a stable neutral radical often used as a spin label. ${ }^{[42]}$ Recently, the similarity between exchange interactions of biradicals including NN unit(s) and electron transport through molecular wires has been pointed out. ${ }^{[43-47]}$ This intimate relationship was deduced from the almost identical exponential attenuation via electron tunneling ${ }^{[48]}$ in both the exchange interaction and molecular conduction through molecular wires. Electron spin resonance (ESR) in the solution phase is ultrasensitive for observing exchange interactions in NN biradicals. ${ }^{[43,44,49-51]}$ Because obtaining single crystals of $\pi$-dimers of conjugated oligomers is difficult, solution phase measurement based on the NN spin label is advantageous.

In the present study, NN radical-substituted conjugated oligomers, the radical cations of which were expected to show $\pi$ dimerization abilities, were designed and synthesized. The compounds chosen were dithienylpyrrole (DTP) derivatives with 
methoxy substituents at the inner $\beta$-position of the thiophene rings, ${ }^{[52]}$ denoted DTP-NN', a diethylpropylenedioxythiophene (ProDOT-Et)-inserted derivative DTP-P-NN', and an ethylenedioxythiophene (EDOT) and ProDOT co-oligomer, ${ }^{[38]}$ $\mathbf{E}_{2} \mathbf{P}_{2}-\mathbf{N N}$. To analyze the possibility of using oligomer radical cation $\pi$-dimers as molecular wires, their electronic and magnetic properties, determined using the NN spin label, are described based on ESR experiments and density functional theory (DFT) calculations.

$$
\text { (1) }
$$

\section{Results and Discussion}

\section{Molecular design and synthesis}

To efficiently synthesize NN-substituted oligomer radical cations, the oxidation potential of the oligomer moiety should be lower than that of the NN unit. ${ }^{[3]}$ Alkyl-substituted oligothiophenes with moderate molecular lengths (3-5 rings), which have typically been used to study $\pi$-dimers, have similar oxidation potentials $\left(0.3-0.4 \mathrm{~V} \text { vs. } \mathrm{Fc} / \mathrm{Fc}^{+}\right)^{[25]}$ to that of aryl-NN $\left(\sim 0.37 \mathrm{~V}\right.$ vs. $\left.\mathrm{Fc} / \mathrm{Fc}^{+}\right) .^{[53]}$ Therefore, the DTP derivatives and $\mathrm{E}_{2} \mathrm{P}_{2}$ cooligomer were chosen because they have lower oxidation potentials $(-0.1-0.0 \mathrm{~V}$ vs. $\left.\mathrm{Fc} / \mathrm{Fc}^{+}\right)^{[38,52]}$ than that of the $\mathrm{NN}$ unit.

The conventional synthetic route to NN-substituted $\pi$-systems involves the oxidation of an $N, N$-dihydroxy-imidazolizine precursor at the final step. ${ }^{[42]}$ However, when a target $\pi$-system has a relatively low oxidation potential, oxidation of the precursor can cause undesired side reactions on the $\pi$-system. Recently, an efficient $\mathrm{Pd}$-catalyzed cross-coupling of [Au(I)(NN-2-ido) $\left.\left(\mathrm{PPh}_{3}\right)\right]$ (NN-Au) and various iodides of $\pi$-systems was developed ${ }^{[54]}$ to overcome this problem. Therefore, this method was used to prepare the target $\mathrm{NN}$-substituted oligomers in the present study.

As shown in Scheme 1, DTP 1 was mono-capped with a methylthio group to give $\mathbf{2}$. lodination of $\mathbf{2}$ under basic conditions, followed by cross-coupling with NN-Au gave DTP-NN: Next, ProDOT-Et 3 was mono iodinated to afford 4 . The Stille coupling of tributylstannylated $\mathbf{2}$ and $\mathbf{4}$ was used to synthesize precursor oligomer $\mathbf{5}$. lodination of $\mathbf{5}$ via lithiation proceeded in $\sim 67 \%$ yield (estimated by ${ }^{1} \mathrm{H}$ NMR) and the cross-coupling of NN-Au with the crude iodide afforded DTP-P-NN ${ }^{*}$. The Stille coupling of the iodide of ProDOT-Et dimer $\mathbf{6}$ with tributylstannylated EDOT dimer afforded 8, which was iodinated and then cross-coupled with NNAu using the same method to afford $\mathbf{E}_{2} \mathbf{P}_{2}-\mathbf{N N}^{*}$.

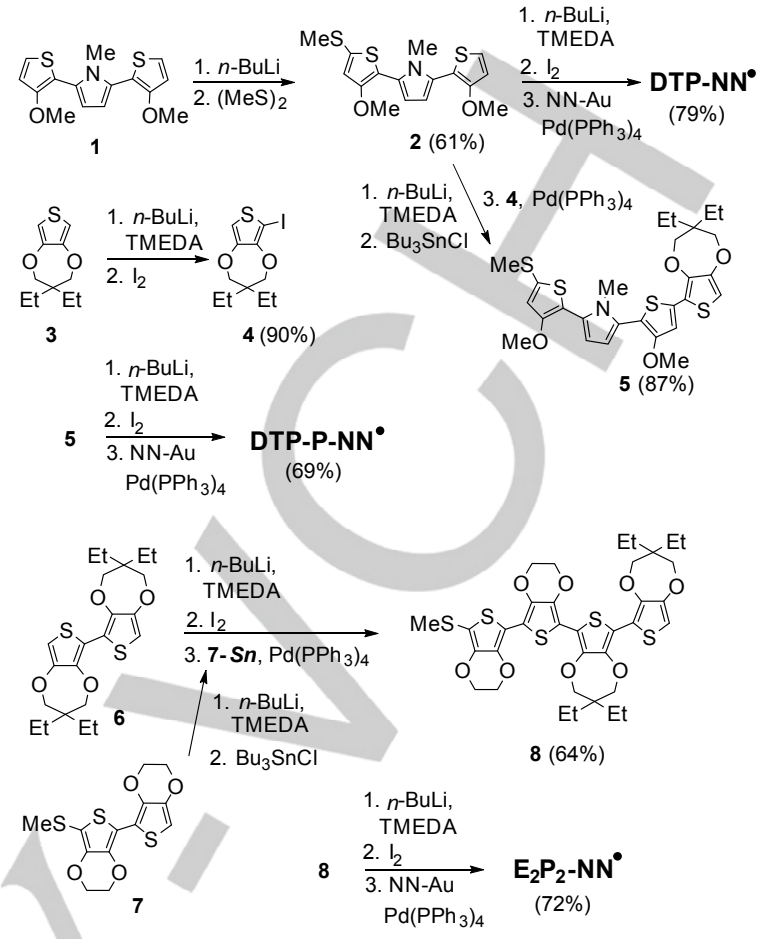

Scheme 1. Synthesis of DTP-NN', DTP-P-NN', and $\mathbf{E}_{2} \mathbf{P}_{2}-\mathbf{N N}^{*}$

\section{Cyclic voltammetry}

The redox properties of DTP-NN', DTP-P-NN', and $\mathbf{E}_{2} \mathbf{P}_{\mathbf{2}}-\mathbf{N N}^{\bullet}$ were investigated by cyclic voltammetry $(\mathrm{CV})$ in dichloromethane (DCM) using tetrabutylammonium hexafluorophosphate $\left(\mathrm{TBAPF}_{6}\right)$ as the electrolyte. As shown in Figure 1, the compounds all showed reversible three-step one-electron oxidations. The half wave potentials for DTP-NN ${ }^{*}$ were +0.06 , +0.42 , and $+0.72 \mathrm{~V}$ vs. $\mathrm{Fc} / \mathrm{Fc}^{+}$, respectively. The first oxidation potential was apparently lower than that of the $\mathrm{NN}$ unit, and comparable with that of DTP with two methylthio end-capping groups $\left(-0.01 \mathrm{~V}\right.$ vs $\left.\mathrm{Fc} / \mathrm{Fc}^{+}\right) .{ }^{[52]}$ Therefore, the first step was considered to be radical cation formation in the DTP moiety. The slight increase in the first oxidation potential of DTP-NN compared with that of bis(methylthio)DTP was thought to be caused by the electron-withdrawing character of the NN unit. In the case of DTP-P-NN', the oxidation potentials $(-0.01,+0.22$, and $+0.51 \mathrm{~V}$ vs. $\mathrm{Fc} / \mathrm{Fc}^{+}$) were lower than those of DTP-NN' due to the $\pi$-extended structure. Similarly, the oxidation potentials of $\mathrm{E}_{2} \mathrm{P}_{2}-\mathrm{NN}^{\bullet}$ were $-0.05,+0.25$, and $+0.51 \mathrm{~V}$ vs. $\mathrm{Fc} / \mathrm{Fc}^{+}$, respectively. Both the first and second oxidation potentials in DTP-P-NN ${ }^{\cdot}$ and $\mathbf{E}_{2} \mathbf{P}_{2}-\mathbf{N N}^{\cdot}$ were apparently lower than those of the $\mathrm{NN}$ unit, suggesting that the first and second steps were radical cation and dication formations in the oligomer moieties, respectively. In practice, these steps were assigned as the formation of the radical cation and dication, as described in the following chemical oxidation. 


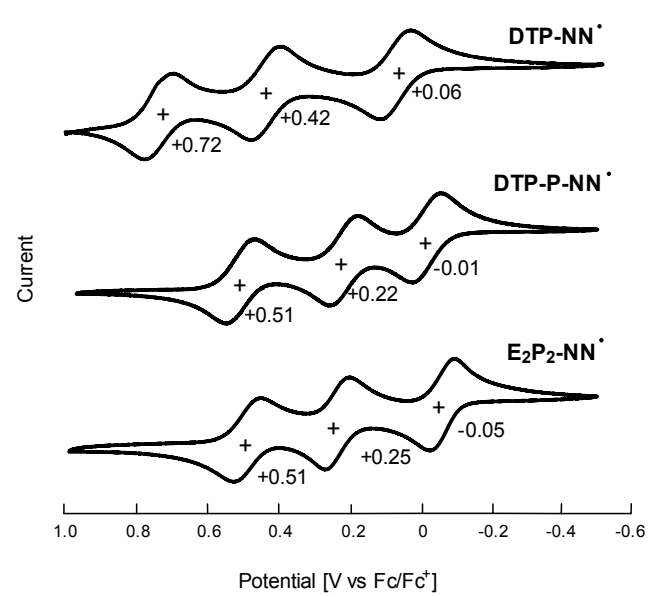

Figure 1. CV of DTP-NN', DTP-P-NN', and $\mathbf{E}_{2} \mathbf{P}_{\mathbf{2}}-\mathbf{N N}^{*}$ in $\mathrm{DCM}$ (supporting electrolyte: $0.1 \mathrm{M} \mathrm{TBAPF}_{6}$; scan rate: $50 \mathrm{mV} \mathrm{s}^{-1}$ ). Values shown are half wave oxidation potentials.

\section{Spectral properties of oxidized monomers}

Chemical oxidation of the $\mathrm{NN}$-substituted oligomers was conducted at room temperature in air. We chose magic blue $\left(\left(\mathrm{BrC}_{6} \mathrm{H}_{4}\right)_{3} \mathrm{~N}^{+} \mathrm{SbCl}_{6}^{-}\right)$as the oxidant for DTP-NN', which had higher oxidation potentials than the other NN-substituted oligomers. The UV-Vis-NIR absorption spectrum of the resulting green solution in DCM (Figure S1 in the Supporting Information (SI)) showed similar absorption bands (609 and $928 \mathrm{~nm}$ ) to those of the bis(methylthio)DTP radical cation (578 and $878 \mathrm{~nm}$ ), ${ }^{[52]}$ indicating that the DTP moiety was oxidized to give an NN radicalsubstituted radical cation (DTP) ${ }^{-+} \mathbf{N N}^{*}$, albeit not very stable species. Unfortunately, the spectral change corresponding to the $\pi$-dimer formation at low temperatures was quite limited, with most (DTP) ${ }^{-+}-\mathrm{NN}^{*}$ remaining as a monomer, even near the DCM freezing point. This result was in sharp contrast to the quantitative $\pi$-dimer formation observed for the bis(methylthio)DTP radical cation under similar conditions. ${ }^{[52]}$ In addition to the possible steric hindrance of NN units, the electron-withdrawing NN unit probably increased the positive charge in the DTP radical cation moiety, which would enhance electrostatic repulsion between the oligomer moieties.

Next, chemical oxidation of DTP-P-NN was performed using $\mathrm{AgSbF}_{6}$ in $\mathrm{DCM}$. As shown in Figure $2 \mathrm{a}$, oxidation with one equivalent of $\mathrm{AgSbF}_{6}$ gave two absorption bands ( $\lambda_{\max } ; 625$ and $1042 \mathrm{~nm}$ ). These absorption bands were similar to the HOMO SOMO and SOMO-LUMO transitions of oligothiophene radical cations $^{[25]}$ and related thiophene-pyrrole mixed oligomers. ${ }^{[52]}$ Therefore, the spectrum can be assigned as a biradical cation (DTP-P) ${ }^{++}-\mathbf{N N}$ : When further oxidation was conducted with one additional equivalent of $\mathrm{AgSbF}_{6}$, the resultant spectrum changed to contain only one intense absorption band $\left(\lambda_{\max } ; 797 \mathrm{~nm}\right)$ which resembled that of oligothiophene dications ${ }^{[25,55]}$ and related thiophene-pyrrole mixed oligomers. ${ }^{[56]}$ Therefore, the second oxidation was also considered to take place at the oligomer moiety rather than the $\mathrm{NN}$ radical, resulting in the $\mathrm{NN}$ radicalsubstituted dication (DTP-P) ${ }^{2+}-\mathbf{N N}^{*}$. Similarly, $\mathbf{E}_{2} \mathbf{P}_{\mathbf{2}}-\mathbf{N N}^{*}$ (Figure $2 b)$ was transformed into biradical cation $\left(E_{2} P_{2}\right)^{-+}-N^{*}\left(\lambda_{\text {max }} ; 713\right.$ and $1216 \mathrm{~nm})$ and radical dication $\left(\mathbf{E}_{2} \mathbf{P}_{2}\right)^{2+}-\mathbf{N N} \cdot\left(\lambda_{\max } ; 854 \mathrm{~nm}\right)$ using one and two equivalents of $\mathrm{AgSbF}_{6}$, respectively.

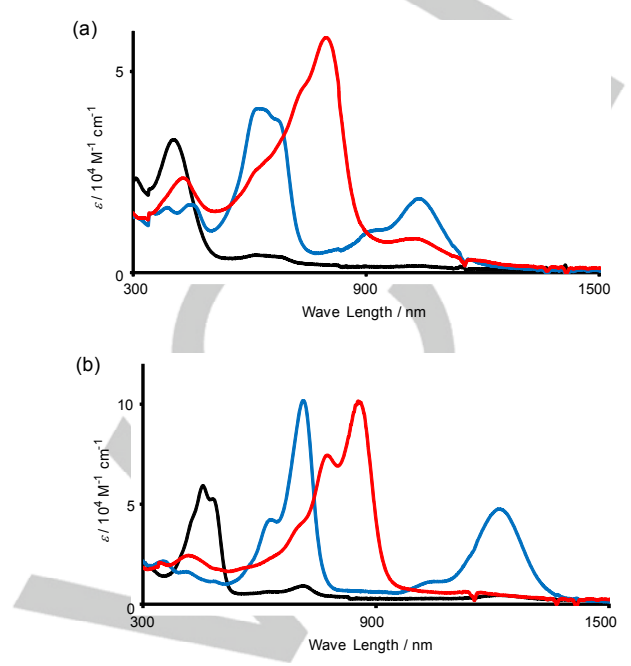

Figure 2. UV-vis-NIR spectra of (a) DTP-P-NN' and (b) $\mathbf{E}_{2} \mathbf{P}_{\mathbf{2}}-\mathbf{N N}^{\cdot}$ and their oneand two-electron oxidized species in DCM. Black, blue, and red lines denote neutral species, biradical cations, and radical dications, respectively.

The ESR spectra of neutral radicals DTP-NN (Figure S2a in SI), DTP-P-NN・ (Figure 3a), and $\mathbf{E}_{2} \mathbf{P}_{\mathbf{2}}-\mathbf{N N}^{*}$ (Figure $3 \mathrm{~b}$ ) measured at room temperature showed five equally separated lines ascribed to the hyperfine coupling of two equivalent nitrogen nuclei in the NN unit. ${ }^{[42]}$ The $g$-factor and the isotropic hyperfine coupling constant were $g=2.006$ and $A / g \mu_{\mathrm{B}}=0.76 \mathrm{mT}$ for three radicals, respectively. The ESR spectra after the two-electron oxidations of DTP-P-NN' and $\mathbf{E}_{2} \mathbf{P}_{2}-\mathbf{N N}^{*}$ with $\mathrm{AgSbF}_{6}$ in $\mathrm{DCM}$ showed similar five-line signals ((DTP-P) ${ }^{2+}-\mathrm{NN}^{*} ; A / g \mu_{\mathrm{B}}=0.74 \mathrm{mT}, g=2.006$ (Figure 3c)) and $\left(\left(\mathbf{E}_{2} \mathbf{P}_{2}\right)^{2+}-\mathbf{N N} ; A / g \mu_{\mathrm{B}}=0.74 \mathrm{mT}, g=2.006\right.$ (Figure $3 \mathrm{~d})$ ), clearly indicating that the generated species retained the $\mathrm{NN}$-radical character with closed-shell nature of the dicationic oligomer moiety.

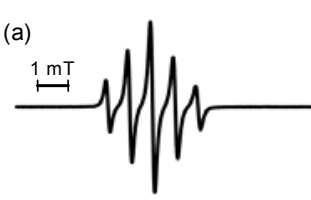

(c)

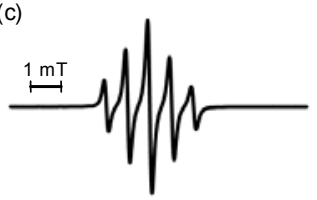

(b)
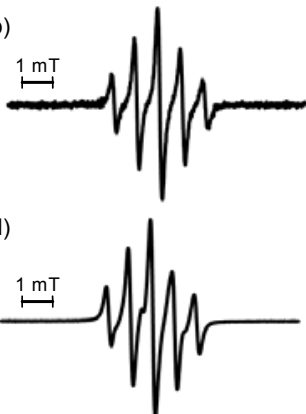

Figure 3. ESR of (a) DTP-P-NN', (b) $\mathbf{E}_{2} \mathbf{P}_{\mathbf{2}}-\mathbf{N N}^{*}$, (c) (DTP-P) ${ }^{2+}-\mathbf{N N}^{*}$, and (d) $\left(\mathrm{E}_{2} \mathrm{P}_{2}\right)^{2+}-\mathrm{NN}^{\bullet}$ in $\mathrm{DCM}$ at room temperature.

In the case of biradical cations (DTP-P) ${ }^{*+}-\mathbf{N N}^{\bullet}$ and $\left(\mathrm{E}_{2} \mathbf{P}_{2}\right)^{-+}-\mathbf{N N}$, the ESR signal showed a characteristic change, as shown in Figure 4 (see Figure S2b in SI for one-electron oxidized (DTP)- 
$\mathrm{NN}^{*}$ ). Simple five-line splittings with equal spacings of $A / g \mu_{\mathrm{B}} \sim$ $0.75 \mathrm{mT}$, as typically found for NN radicals (Figure 3 ), disappeared, with the spectra exhibiting splitting with apparently half the coupling, $A / 2 g \mu_{\mathrm{B}} \sim 0.37 \mathrm{mT}$. This change should be caused by an intramolecular exchange interaction $\left(J_{\text {intra }}\right)$ between the oligomer radical cation and NN radical moieties. The ESR spectra were analyzed by simulating spectral patterns using the spin Hamiltonian $H^{\mathrm{m}}$ (superscript $\mathrm{m}$ denotes monomer) for the aggregation-free monomer of the biradical cations (equation (1))

$$
\begin{aligned}
H^{\mathrm{m}}= & -2 J_{\text {intra }}^{\mathrm{m}} \mathbf{S}_{1} \cdot \mathbf{S}_{2}+\mu_{\mathrm{B}} B\left(g_{1} S_{1}^{\mathrm{z}}+g_{2} S_{2}^{\mathrm{z}}\right) \\
& +A^{\mathrm{m}} S_{1}^{\mathrm{z}}\left(I_{1 \mathrm{a}}^{\mathrm{z}}+I_{1 \mathrm{~b}}^{\mathrm{z}}\right)
\end{aligned}
$$

where the first term represents the Heisenberg-type exchange interaction between the 1/2-spins in the NN radical $\left(\mathbf{S}_{1}\right)$ and the oligomer radical cation $\left(\mathbf{S}_{2}\right)$ moieties with exchange parameter $\mathrm{Jm}_{\text {intra. }}$. The second and third terms denote the electronic Zeeman interaction with magnetic field $B$, and the hyperfine coupling for nitrogen atoms of spin $I=1$ with coupling constant $A^{\mathrm{m}}$ in $\mathrm{NN}$, respectively.

(a)

(i) obs.

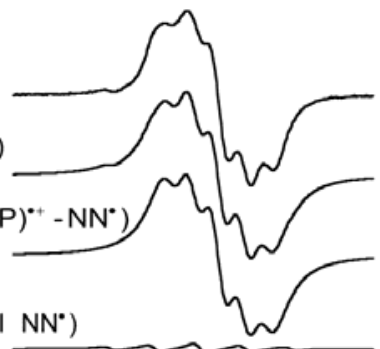

(iv) sim. (residual $\mathrm{NN}^{*}$ )

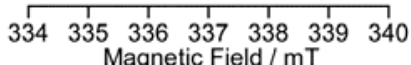

(b)

(i) obs.

(ii) $\operatorname{sim}$.((iii)+(iv))

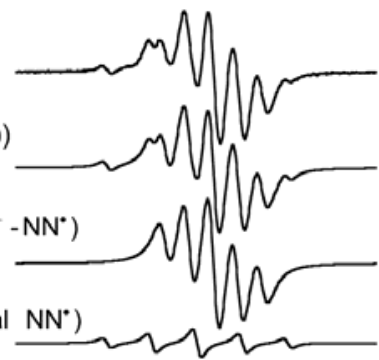

(iv) $\operatorname{sim}$. (residual $\mathrm{NN}^{*}$ )

(iii) $\operatorname{sim} .\left(\left(\mathrm{E}_{2} \mathrm{P}_{2}\right)^{*+}-\mathrm{NN}^{*}\right)$

$\begin{array}{lllllll}334 & 335 & 336 & 337 & 338 & 339 & 340\end{array}$ Magnetic Field / mT

Figure 4. Observed and simulated ESR spectra of (a) (DTP-P) ${ }^{*+}-\mathbf{N N}^{\bullet}(0.70 \mathrm{mM}$ (precursor), $T=293 \mathrm{~K}, v=9.461536 \mathrm{GHz}),(b)\left(\mathbf{E}_{2} \mathbf{P}_{2}\right)^{++}-\mathbf{N N}^{*}(0.25 \mathrm{mM}$ (precursor), $T=290 \mathrm{~K}, v=9.461752 \mathrm{GHz}$ ); (i) observed in DCM, (ii) simulated (total), (iii) simulated (biradical cation monomer), (iv) simulated (residual NN).

The two spins, $\mathbf{S}_{1}$ and $\mathbf{S}_{2}$, are assumed to have distinct $g$ factors, $g_{1}$ and $g_{2}$. A schematic of the spin Hamiltonian, $H^{\mathrm{m}}$, is shown in Figure 5. The hyperfine splitting pattern of an exchangecoupled spin system is known to reflect the relative magnitudes of the exchange and hyperfine interactions, ${ }^{[57]}$ which were $\left|\mathrm{Jm}_{\text {intral }}\right|$ and $\left|A^{\mathrm{m}}\right|$ for the biradical cations (DTP-P) ${ }^{*+}-\mathbf{N N}^{\bullet}$ and $\left(\mathbf{E}_{2} \mathbf{P}_{2}\right)^{*+}-\mathbf{N N}^{\cdot}$. The position of the central resonance field, $B^{\mathrm{c}}$, for a series of hyperfine transitions is governed by the exchange-averaged $g$ factor, which reflects the relative magnitudes of the exchange $\left|J^{\mathrm{intra}}\right|$ and the difference in the Zeeman interactions, $\Delta g \mu_{\mathrm{B}} B^{\mathrm{c}}=$ $\left(g_{1}-g_{2}\right) \mu_{\mathrm{B}} B^{\mathrm{c}}$. The spectral patterns for the two-spin system of the biradical cations were clarified from simulations ${ }^{[58]}$ based on $H^{\mathrm{m}}$ (See Figure $\mathrm{S} 3$ in $\mathrm{SI}$ ) as a function of the relative magnitudes of $\left|J_{\text {intra }}\right| /|A|$ and $\left|J_{\text {intra }}\right| / \Delta g \mu_{\mathrm{B}} B^{\mathrm{c}}$. A symmetric five-line splitting with a spacing of $A / 2 g \mu_{\mathrm{B}} \sim 0.37 \mathrm{mT}$ centered at $g_{\mathrm{av}}=\left(g_{1}+g_{2}\right) / 2$ for the biradical cation appeared when two conditions, namely $\mid J^{\mathrm{m}}$ intra $/ A^{\mathrm{m}} \mid$ $>100$ and $\left|J^{m_{i n t r a}} / \Delta g \mu_{\mathrm{B}} B^{\mathrm{C}}\right|>100$, were fulfilled. The observed spectra for (DTP-P) ${ }^{++}-\mathrm{NN}^{*}$ were reproduced by superposing the signals from the biradical cation and a residual NN radical. The residual $\mathrm{NN}$ radical was assigned to a decomposed species only at the radical cation moiety, and/or the neutral or dicationic species, which could not be avoided in the oxidation process. In the simulation of the superposed spectra, the doubly integrated intensity DI of ESR spectra was assumed to be proportional to the static paramagnetic susceptibility $\chi$ of the paramagnetic entities as given by equation (2),

$D I($ biradical cation $): D I($ residual $\mathrm{NN})=\chi($ biradical cation $): \chi($ residual $\mathrm{NN})$

$$
=\frac{p N_{\mathrm{A}} g_{\mathrm{av}}^{2} \mu_{\mathrm{B}}^{2}}{k_{\mathrm{B}} T} \frac{2}{3+\exp \left(-2 J_{\text {intra }}^{\mathrm{m}} / k_{\mathrm{B}} T\right)}: \frac{(1-p) N_{\mathrm{A}} g_{1}^{2} \mu_{\mathrm{B}}^{2}}{4 k_{\mathrm{B}} T}
$$

where $p$ represents the purity. The intensity of the biradical cation is given by the thermal equilibrium of the triplet and singlet states with an energy gap of $\left|2 J^{m_{\text {intral }}}\right|$, while the intensity of residual $N N$ simply obeys the Curie law. The superposed spectra of the two components are shown in Figure 4. The parameters used in the simulation for (DTP-P) ${ }^{-+}-\mathrm{NN}^{\bullet}$ were $A^{\mathrm{m}} / \mathrm{g}_{1} \mu_{\mathrm{B}}=0.790 \mathrm{mT}, \mathrm{Jm}_{\text {intral }} / \mathrm{k}_{\mathrm{B}}=$ $+300 \mathrm{~K}$ (the lower limit obtained from quantitative ESR, see below), $g_{1}=2.0053, g_{2}=2.0017, \Delta B=0.420 \mathrm{mT}$ (line width for a single Lorentzian), and $p=0.96$ for the biradical cation, and $A / g_{1} \mu_{\mathrm{B}}=0.755 \mathrm{mT}, g_{1}=2.0053$, and $\Delta B=0.230 \mathrm{mT}$ for residual NN. The ratio of the double-integrated intensity was $D I(\text { (DTP-P) })^{++}$ $\left.\mathrm{NN}^{*}\right): D I($ residual $\mathrm{NN})=0.984: 0.016$. The parameters for $\left(\mathbf{E}_{2} \mathbf{P}_{2}\right)^{-+} \mathbf{N N}^{\cdot}$ were $A^{\mathrm{m}} / g_{1} \mu_{\mathrm{B}}=0.803 \mathrm{mT}, \mathrm{Jm}_{\text {intral }} / \mathrm{k}_{\mathrm{B}}=+300 \mathrm{~K}, g_{1}=$ 2.0059, $g_{2}=2.0027, \Delta B=0.235 \mathrm{mT}$, and $p=0.85$ for the biradical cation, and $A / g_{1} \mu_{\mathrm{B}}=0.755 \mathrm{mT}, g_{1}=2.0059$, and $\Delta B=0.150 \mathrm{mT}$ for residual NN. The ratio of the double-integrated intensity was $D I\left(\left(\mathbf{E}_{2} \mathbf{P}_{2}\right)^{*+} \mathbf{- N N}\right): D I($ residual NN $)=0.935: 0.065$. The $g$-factors, $g_{1}(2.0053-2.0059)$ and $g_{2}(2.0017-2.0027)$, fell within the typical values of NN radicals ${ }^{[59,60]}$ and radical cations of oligothiophene with alkoxy substituents, ${ }^{[55]}$ respectively. Simulations indicated that $\mid J^{\mathrm{m}}$ intra $/ A^{\mathrm{m}} \mid$ and $\left|J^{\mathrm{m}} \mathrm{intra} / \Delta g \mu_{\mathrm{B}} B^{\mathrm{C}}\right|$ were larger than 100 , showing that the aggregation-free monomer of the biradical cations had a strong $\left|J_{\text {intra }}\right| / k_{\mathrm{B}}(\geq 0.1 \mathrm{~K})$ in both (DTP-P) $)^{-+}-\mathbf{N N}^{\cdot}$ and $\left(\mathbf{E}_{2} \mathbf{P}_{2}\right)^{-+}-\mathbf{N N}^{\cdot}$. 
Table 1. Total energies $E$ and $\left\langle\mathrm{S}^{2}\right\rangle$ values for the BS singlet and high spin (Triplet) states and intramolecular exchange ilnteraction $\mathrm{J}^{\mathrm{m}_{\text {intra }}}$ of (DTP-P) ${ }^{\cdot+}$-NN ${ }^{\bullet}$ and $\left(\mathrm{E}_{2} \mathrm{P}_{2}\right)^{++}-\mathrm{NN} \cdot$ monomers.

\begin{tabular}{|c|c|c|c|c|c|c|}
\hline compd & Method $^{[a]}$ & $E_{\mathrm{BS}} /$ hartree & $E_{T} /$ hartree & $\left\langle S^{2}\right\rangle_{\mathrm{BS}}$ & $\left\langle S^{2}\right\rangle_{T}$ & $J^{m_{i n t r a}} k_{B}{ }^{-1} / \mathrm{K}$ \\
\hline \multirow[t]{3}{*}{$(\mathrm{DTP}-\mathrm{P})^{\cdot+}-\mathrm{NN}^{\cdot}$} & B97D3(BJ) & -3527.71759 & -3527.71760 & 0.8027 & 2.0275 & +5 \\
\hline & B3LYP & -3528.87011 & -3528.87355 & 1.0709 & 2.1079 & +1048 \\
\hline & M06-2X & -3527.92641 & -3527.92949 & 1.1298 & 2.1878 & +918 \\
\hline \multirow[t]{3}{*}{$\left(E_{2} P_{2}\right)^{\cdot+}-N^{\cdot}$} & B97D3(BJ) & -4481.97465 & -4481.97514 & 0.8137 & 2.0258 & +130 \\
\hline & B3LYP & -4483.45370 & -4483.45793 & 1.0672 & 2.1008 & +1292 \\
\hline & M06-2X & -4482.27467 & -4482.27920 & 1.1305 & 2.1894 & +1350 \\
\hline
\end{tabular}

[a] With 6-31G(d) basis set.

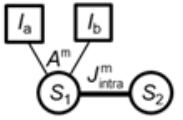

(ii)

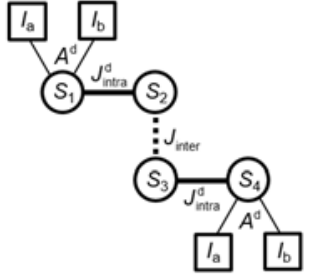

(iii)

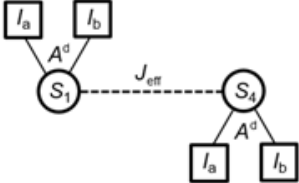

Figure 5. Schematic diagram of spin Hamiltonians. (i) Two-spin system for the cationic biradical monomer (equation (1)). (ii) Four-spin system for the $\pi$-dimer (equation (4)). (iii) Effective two-spin system (equation (5)). Circles denote the $S=1 / 2$ spins, which are coupled with squares representing the $I=1$ spins of nitrogen nuclei by hyperfine interactions $A^{\mathrm{m}}$ and $A^{\mathrm{d}}$ (thin solid lines). Thick solid lines represent intramolecular exchange interactions $J^{\mathrm{m}}$ intra and $J^{\mathrm{d}}$ intra, while the dotted line denotes intermolecular exchange interaction $J_{\text {inter. Dashed line }}$

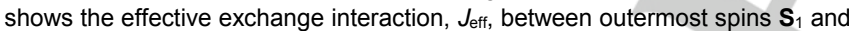
$\mathbf{S}_{4}$.

\section{DFT calculations of oxidized monomers}

Because of $\pi$-dimer formation, as shown below, it was difficult to experimentally determine the $\mathrm{Jm}_{\text {intra }}$ values with ESR in a lowtemperature matrix. Therefore, the magnitude of the exchange interaction $(J)$ was estimated by DFT calculations according to Yamaguchi's equation (3). ${ }^{[61]}$

$$
J=\frac{E_{B S}-E_{H S}}{\left\langle S^{2}>_{H S^{-}}<S^{2}\right\rangle_{B S}}
$$

where $E$ and $\left\langle S^{2}\right\rangle$ are the total energy and expectation value of total $S^{2}$, respectively, with subscripts BS and HS denoting the singlet with broken symmetry (BS) method and high spin state, respectively. The B3LYP method is frequently used to estimate $J$ values. ${ }^{[47,62,63]}$ However, in general, the method cannot be applied to the full optimization of ion radical $\pi$-dimers without dispersion or long range correction. ${ }^{[35,64]}$ Therefore, B97D3(BJ) (recommended for the geometry optimization of ion radical dimers $^{[64]}$ ) and M06-2X (used for $\pi$-dimers in previous studies $^{[35,38,52]}$ ) and B3LYP with the $6-31 \mathrm{G}(\mathrm{d})$ basis set were used to estimate $\mathrm{Jm}_{\text {intra. }}$. The basis set may be small for accurate prediction of properties, but it would be sufficient for obtaining qualitative trends. The results are summarized in Table 1.

These methods all showed that the triplet states were more stable than the singlet states, resulting in positive $\mathrm{J}^{\mathrm{m}}$ intra values. These positive $\mathrm{Jm}_{\text {intra }}$ values can be explained by the spin polarization mechanism. As shown in Figure S4 in SI, the brokensymmetry singlet states of both (DTP-P) ${ }^{++}-\mathbf{N N}^{\cdot}$ and (E2P2) ${ }^{-+}-\mathbf{N N}^{\cdot}$ showed an unfavorable parallel spin pair at the position across the $\alpha$-carbon of terminal thiophene ring and its connecting carbon in the NN unit, while the favorable antiparallel alignment was found at the same position in the triplet state. The B3LYP and M06-2X methods gave comparable $\mathrm{J}_{\text {intra }}$ values for both (DTPP) ${ }^{\cdot+}-\mathbf{N N}^{\cdot}$ and (E2P2) $)^{\cdot+}-\mathbf{N N}^{\bullet}$. In contrast, the $\mathrm{J}^{\mathrm{intra}}$ values obtained using the B97D3 method were much smaller. M06-2X is a hybrid DFT with Hartree-Fock exchange similar to B3LYP, while B97D3 is a "pure" DFT. Pure DFT methods tend to avoid symmetry breaking, but hybrid DFT methods do not. ${ }^{[64,65]}$ In practice, the $\left\langle\mathrm{S}^{2}\right\rangle_{\text {BS }}$ values from B97D3 for both (DTP-P) ${ }^{-+}-\mathbf{N N}^{\cdot}$ and $\left(\mathbf{E}_{2} \mathbf{P}_{2}\right)^{-{ }_{-}}$ $\mathbf{N N}^{*}$ were around 0.8 , which was smaller than the value of 1 expected for species with high singlet diradical character. The diradical index based on natural orbital LUMO occupation numbers (NOON) ${ }^{[66]}$ were 0.546 and 0.559 , respectively. The small diradical character (i.e., larger covalent character) in the BS singlet states would cause some stabilization energy, resulting in a narrow singlet-triplet energy gap, whereas the effect of the denominator in equation (3) was small. Therefore, the B97D3 method underestimated the $\mathrm{J}^{\mathrm{m}}$ intra values. In contrast, as a related example of an NN-radical-substituted radical cation, a 5,10diphenyl-5,10-dihydrophenazine derivative showed a relatively large $\mathrm{Jm}_{\text {intra }}$ value $(+700 \mathrm{~K}$ from simulation of the SQUID measurement; $+987 \mathrm{~K}$ from B3LYP/6-31G(d) calculations based on the X-ray structure). ${ }^{[67]}$ The calculated $J \mathrm{~m}_{\text {intra }}$ with B3LYP was slightly overestimated. These comparisons suggested that the actual $J \mathrm{~m}_{\text {intra }}$ values were within the range between the values calculated using the pure and hybrid DFT methods $(+5$ to +1000 $\mathrm{K}$ for (DTP-P) ${ }^{++}-\mathbf{N N}^{*}$ and +130 to $+1300 \mathrm{~K}$ for $\left.\left(\mathrm{E}_{2} \mathbf{P}_{2}\right)^{++}-\mathbf{N N}^{*}\right)$. However, the hybrid DFTs would give $J^{\mathrm{m}}$ intra values closer to the actual values than pure DFT. In fact, the value of (DTP-P) $)^{-+}-\mathbf{N N}^{*}$ with B97D3 method was inconsistent with the experimental result obtained from quantitative ESR as shown below.

\section{Spectral properties of $\pi$-dimers}

In contrast to (DTP) ${ }^{*+}-\mathbf{N N}^{*}$, which showed limited $\pi$-dimer formation, (DTP-P) $)^{++}-\mathbf{N N}^{\cdot}$ and $\left(\mathbf{E}_{2} \mathbf{P}_{2}\right)^{+{ }^{+}}-\mathbf{N N}^{\cdot}$ showed almost quantitative $\pi$-dimer formation. As shown in Figure 6 , the diagnostic spectral change for the formation of oligomer radical 
cation $\pi$-dimer, comprising three absorption bands due to a HOMO-LUMO transition in the $\pi$-dimer with blue-shifted original bands for the radical cation moiety, ${ }^{[29,35]}$ was observed in DCM at low temperatures. The longest absorption maxima were assigned to HOMO-LUMO transitions of the $\pi$-dimer and found at $1040 \mathrm{~nm}$ for (DTP-P-NN $)_{2}{ }^{2+}$ and $\sim 1270 \mathrm{~nm}$ for $\left(\mathrm{E}_{2} \mathbf{P}_{\mathbf{2}}-\mathrm{NN}^{*}\right)_{2}{ }^{2+}$. The broader band of the HOMO-LUMO transition for $\left(\mathrm{E}_{2} \mathbf{P}_{2}-\mathrm{NN}^{*}\right)_{2}{ }^{2+}$ was attributed to the less efficient SOMO-SOMO overlap due to the all-trans conformation of the EDOT-ProDOT co-oligomer moiety, ${ }^{[38]}$ whereas (DTP-P-NN ${ }^{*}{ }^{2+}{ }^{2+}$ would have better overlap owing to the all-cis conformation in the DTP moiety. ${ }^{[52]}$

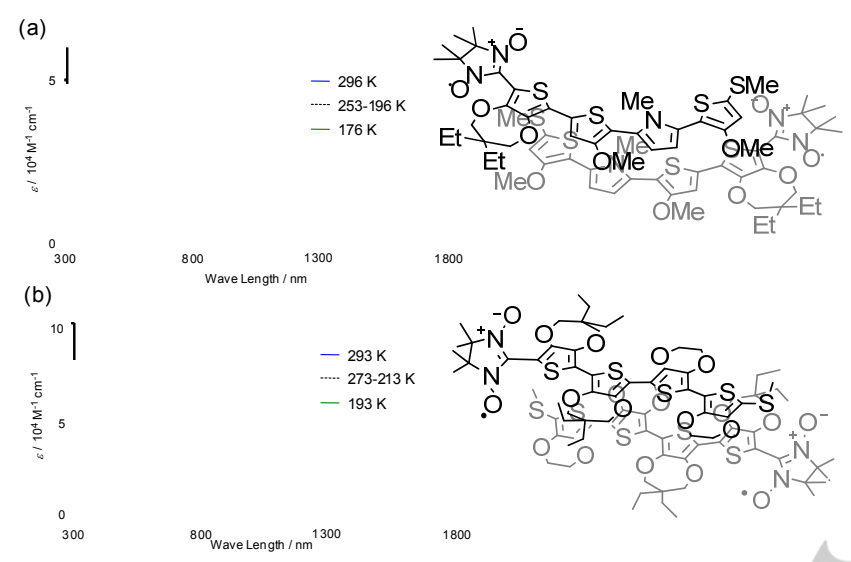

Figure 6. Variable temperature UV-vis-NIR spectra of (a) (DTP-P) ${ }^{\cdot+}-N^{*} \cdot(0.29$ $\mathrm{mM}$ ((precursor)) and (b) $\left(\mathrm{E}_{2} \mathbf{P}_{2}\right)^{-+}-\mathbf{N N}^{\cdot}(0.23 \mathrm{mM}$ (precursor)) in DCM with schematic structures of their $\pi$-dimers (DTP-P-NN $)_{2}{ }^{2+}$ and $\left(\mathrm{E}_{2} \mathrm{P}_{2}-\mathrm{NN}^{*}\right)_{2}{ }^{2+}$.

ESR spectra of (DTP-P-NN $\left.{ }^{\bullet}\right)_{2}{ }^{2+}$ and $\left(\mathrm{E}_{2} \mathrm{P}_{\mathbf{2}}-\mathrm{NN}^{\bullet}\right)_{2}{ }^{2+}$ taken in DCM at low temperatures are shown in Figure 7. (See Figure S5 in SI for temperature dependence of (DTP-P)*+-NN*). The signals involved nine-line splittings with apparently half the coupling, $A / 2 g \mu_{\mathrm{B}} \sim 0.38 \mathrm{mT}$, at the central field of $g=2.006$. This suggested a strong exchange interaction $\left|J_{\text {intra }}\right|>>|A|$ between $N N$ units, while no contribution was found for the oligomer radical cation units in the ESR spectra, as judged from the $g$-factor. The ESR spectra were simulated using the four-spin Hamiltonian $H^{d}$ (superscript $d$ denotes $\pi$-dimer) for the $\pi$-dimerized biradical cations (DTP-P$\left.\mathrm{NN}^{*}\right)_{2}{ }^{2+}$ and $\left(\mathrm{E}_{2} \mathrm{P}_{2}-\mathrm{NN}^{*}\right) \mathbf{2}^{2+}$, as shown in equation (4),

$$
\begin{aligned}
H^{\mathrm{d}}= & -2 J_{\text {intra }}^{\mathrm{d}}\left(\mathbf{S}_{1} \cdot \mathbf{S}_{2}+\mathbf{S}_{3} \cdot \mathbf{S}_{4}\right)-2 J_{\text {inter }} \mathbf{S}_{2} \cdot \mathbf{S}_{3} \\
& +\mu_{\mathrm{B}} B\left(g_{1} S_{1}^{Z}+g_{2} S_{2}^{Z}+g_{3} S_{3}^{Z}+g_{4} S_{4}^{\mathrm{Z}}\right) \\
& +A^{\mathrm{d}} S_{1}^{\mathrm{Z}}\left(I_{1 \mathrm{a}}^{\mathrm{Z}}+I_{1 \mathrm{~b}}^{\mathrm{Z}}\right)+A^{\mathrm{d}} S_{4}^{\mathrm{Z}}\left(I_{4 \mathrm{a}}^{\mathrm{Z}}+I_{4 \mathrm{~b}}^{\mathrm{Z}}\right)
\end{aligned}
$$

where the terms in the Hamiltonian had the same meanings as described in equation (1). A schematic of the spin Hamiltonian $H^{\mathrm{d}}$ is shown in Figure 5 (ii). The inner two of the four 1/2-spins, $\mathbf{S} 2$ and $\mathbf{S} 3$, represent the oligomer radical cation with $g$-factor $g_{2}=g_{3}$, while the outer two spins, $\mathbf{S} 1$ and $\mathbf{S} 4$, corresponded to the NN radical with $g$-factor $g_{1}=g_{4}$ and carrying nitrogen nuclei of $I=1$. The spectral patterns for the four-spin system mimicking the biradical dimers were clarified using simulations ${ }^{[58]}$ based on $H^{\mathrm{d}}$ as a function of the ratio of $\mathrm{J}^{\mathrm{d}} \mathrm{intra} /\left|\mathrm{J}_{\text {inter }}\right|$ (see Figure $\mathrm{S} 6$ in $\mathrm{SI}$ ), with the pattern of hyperfine splittings and position of the central resonance fields depending on $\mathrm{J}_{\text {intral }} /\left|J_{\text {inter }}\right|$. The weak limit of the intramolecular exchange interaction, $J^{\mathrm{d}}$ intra ||$J_{\text {inter }} \mid \ll<1$, was found to give a five-line splitting with a spacing of $A / g \mu_{\mathrm{B}} \sim 0.75 \mathrm{mT}$ centered at $g_{1} \sim 2.006$, which was typical of NN monoradicals. In contrast, the strong limit of $J^{\mathrm{d}}$ intra $/\left|J_{\text {inter }}\right| \gg 1$ afforded a nine-line splitting with a quarter of the spacing, $A / 4 g \mu_{\mathrm{B}} \sim 0.19 \mathrm{mT}$, centered at $g_{\mathrm{av}}=\left(g_{1}+g_{2}\right) / 2 \sim 2.004$. Between the two extremes was a nine-line pattern with half the spacing of $A / 2 g \mu_{\mathrm{B}} \sim 0.37 \mathrm{mT}$ centered at $g_{1} \sim 2.006$, which was typical of NN biradicals in a triplet state. However, the appearance of the NN biradical-like nine-line pattern was found to require a relatively strong $J_{\text {inter }}$, and the weaker $\left|J_{\text {inter }}\right| / k_{\mathrm{B}} \leq 400 \mathrm{~K}$ did not show the clear nine-line pattern even using any $J^{d}$ intra value (see Figure S6c-e). The biradical-like pattern came to appear when $\left|J_{\text {inter }}\right| / k_{\mathrm{B}}$ was $500 \mathrm{~K}$ associated with $\mathrm{J}_{\text {intra }}$ in the range of $+10 \mathrm{~K} \leq \mathrm{J}^{\mathrm{d}}$ intra $\leq+100 \mathrm{~K}$ (see Figure S6f). In the case of $J_{\text {inter }} / k_{\mathrm{B}}=-1719 \mathrm{~K}$ or $=-1201 \mathrm{~K}$ (both $J_{\text {inter }}$ values from M06-2X calculations, see below), the biradicallike pattern appeared in the range $+100 \mathrm{~K} \leq \mathrm{J}^{\mathrm{d}}$ intra $\leq+400 \mathrm{~K}$ or $+300 \mathrm{~K}$ (see Figure S6a,b).

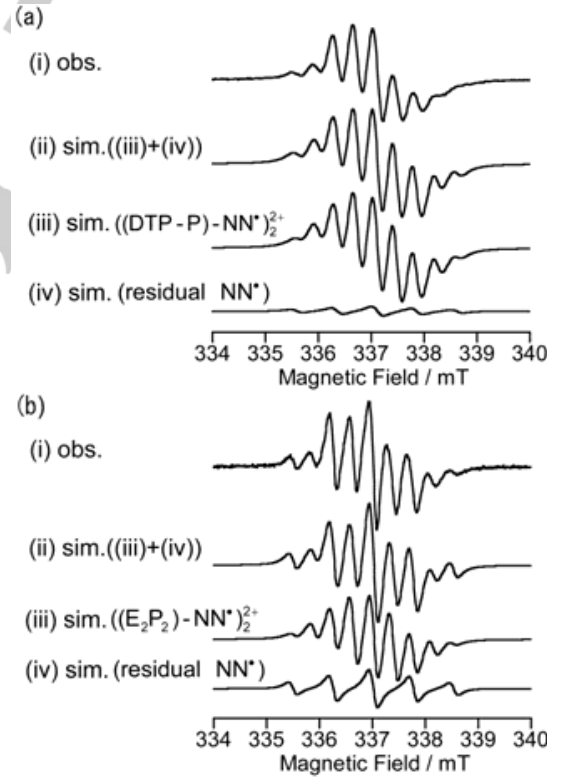

Figure 7. ESR spectra of (a) (DTP-P-NN $)_{2}{ }^{2+}(T=200 \mathrm{~K}, v=9.461729 \mathrm{GHz})$, (b) $\left(E_{2} P_{2}-N^{*}\right)_{2}{ }^{2+}(T=206 \mathrm{~K}, v=9.461812 \mathrm{GHz})$ in DCM (same samples in Figure 4) and their simulated spectra; (i) observed in DCM, (ii) simulated (total), (iii) simulated ( $\pi$-dimers), (iv) simulated (residual $N N$ ).

The observed spectra for the $\pi$-dimers were reproduced by the superposition of signals from the biradical cation and a residual $\mathrm{NN}$ radical, where the purity $p$ was assumed to remain unchanged after dimerization in the low-temperature experiments. The parameters used in the simulation for (DTP-P-NN $)_{2}{ }^{2+}$ were $A^{\mathrm{d}} / g_{1} \mu_{\mathrm{B}}=0.755 \mathrm{mT}, J^{\mathrm{d}}{ }_{\text {intra }} / k_{\mathrm{B}}=+100 \mathrm{~K}$ (overlap region for $500 \mathrm{~K} \leq$ $\left.\left|J_{\text {inter }}\right| / k_{\mathrm{B}} \leq 1719 \mathrm{~K}\right),\left|J_{\text {inter }}\right| / k_{\mathrm{B}}=500 \mathrm{~K}$, (lower limit of $\left|J_{\text {interl }}\right| / k_{\mathrm{B}}$ from the simulation), $g_{1}=g_{4}=2.0053, g_{2}=g_{3}=2.0017, \Delta B=0.24 \mathrm{mT}$, and $p=0.96$ for the biradical cation, and $A^{\mathrm{d}} / g_{1} \mu_{\mathrm{B}}=0.755 \mathrm{mT}, g_{1}=$ 
2.0053 , and $\Delta B=0.23 \mathrm{mT}$ for residual NN. The ratio of the doubleintegrated intensity was $D I\left((\text { DTP-P.NN })^{*}{ }^{2+}\right): D I($ residual $N N)=$ $0.96: 0.04$. The parameters for $\left(\mathbf{E}_{2} \mathbf{P}_{2}-\mathbf{N N}\right)^{*}{ }_{2}{ }^{2+}$ were $A^{\mathrm{d}} / g_{1} \mu_{\mathrm{B}}=$ $0.755 \mathrm{mT}, J^{\mathrm{d}} \mathrm{intra}_{\mathrm{B}} / \mathrm{k}_{\mathrm{B}}=+300 \mathrm{~K},\left|\mathrm{~J}_{\text {inter }}\right| / \mathrm{K}_{\mathrm{B}}=500 \mathrm{~K}, g_{1}=g_{4}=2.0059$, $g_{2}=g_{3}=2.0027, \Delta B=0.20 \mathrm{mT}$, and $p=0.85$ for the biradical cation, and $A / g_{1} \mu_{\mathrm{B}}=0.755 \mathrm{mT}, g_{1}=2.0059$, and $\Delta B=0.170 \mathrm{mT}$ for residual NN. The ratio of the double-integrated intensity was $D I\left(\left(\mathbf{E}_{2} \mathbf{P}_{2}-\mathbf{N N}^{*}\right)_{2}{ }^{2+}\right): D I($ residual $\mathrm{NN})=0.85: 0.15$. The simulation results indicated that the oligomer radical cations in (DTP-P$\left.\mathrm{NN}^{*}\right)_{2}{ }^{2+}$ and $\left(\mathrm{E}_{2} \mathrm{P}_{2}-\mathrm{NN}^{*}\right)_{2}{ }^{2+}$ lost their contributions to the ESR spectra on $\pi$-dimerization, as seen in the $g$-factors, while sizable exchange interactions between the $\mathrm{NN}$ moieties lasted throughout the oligomer cation dimers. If the exchange interactions were vanishing, the resultant hyperfine splitting patterns should have been those expected for single NN monoradicals, namely a five-line splitting with $A / g \mu_{\mathrm{B}} \sim 0.75 \mathrm{mT}$, corresponding to the weak limit of $J^{\mathrm{d}_{\text {intra }}} /\left|J_{\text {inter }}\right|<<1$.

In our previous study, ${ }^{[57]}$ we discussed the spin states and ESR spectra of exchange-coupled multi-spin systems in molecular aggregates of two or more symmetric biradical molecules, each composed of two isostructural monoradical fragments, on the basis of theoretical spin Hamiltonians, such as equation (4). ${ }^{[57]}$ One conclusion obtained for the four-spin system of equation (4) was that the ESR spectral patterns were classified into three regions according to the relative magnitude of $J^{\mathrm{d}}$ intra with respect to $\left|J_{\text {inter }}\right|$ and $A^{d}{ }^{\mathrm{i}}{ }^{[7]}$ When $A^{\mathrm{d}}<<\left|J_{\text {inter }}\right|<J^{\mathrm{d}}$ intra (Case a), all four $1 / 2$ spins contributed to the ESR spectra, and the dimer behaved as a "tetraradical" exhibiting a fourth of the hyperfine spacing $A / 4 g \mu_{\mathrm{B}}$ in the ESR spectra. When $\left|A^{\mathrm{d}}\right| \ll J^{\mathrm{d}}$ intra $<\left|J_{\text {inter }}\right|$ (Case b), the contributions of the inner two 1/2-spins, $\mathbf{S}_{2}$ and $\mathbf{S}_{3}$, to the ESR spectra were lost, but the outer two 1/2-spins, $\mathbf{S}_{1}$ and $\mathbf{S}_{4}$, remained coupled by an effective exchange interaction, giving a biradical-like splitting with half the spacing of $A / 2 g \mu_{\mathrm{B}}$. When $J^{\mathrm{d}}$ intra $\ll\left|A^{\mathrm{d}}\right| \ll\left|J_{\text {inter }}\right|$ (Case c), the outer two 1/2-spins behaved as decoupled monoradicals, exhibiting a simple hyperfine spacing of Alg $\mu_{\mathrm{B}}$.

In Case b, the four-spin system of equation (4) was approximated by the two-spin system (Figure 5 (iii)) as equation (5),

$$
\begin{aligned}
H^{\mathrm{eff}}= & -2 J_{\mathrm{eff}} \mathbf{S}_{1} \cdot \mathbf{S}_{4}+\mu_{\mathrm{B}} B\left(g_{1} S_{1}^{\mathrm{z}}+g_{4} S_{4}^{\mathrm{z}}\right) \\
& +A^{\mathrm{d}} S_{1}^{\mathrm{z}}\left(I_{1 \mathrm{a}}^{\mathrm{z}}+I_{1 \mathrm{~b}}^{\mathrm{z}}\right)+A^{\mathrm{d}} S_{4}^{\mathrm{z}}\left(I_{4 \mathrm{a}}^{\mathrm{z}}+I_{4 \mathrm{~b}}^{\mathrm{z}}\right)
\end{aligned}
$$

where $J_{\text {eff }}$ denotes the effective exchange interaction between the outermost spins. The equivalence between the four-spin system of the dimeric aggregate and the effective two-spin system with $J_{\text {eff }}$ has been rationalized by considering the spin-spin correlation functions and ESR spectral simulations. ${ }^{[57,68]}$ The four-spin system had six eigenstates, two singlet $(S=0)$ states, three triplet $(S=1)$ states, and a quintet $(S=2)$ state. In the range of $J^{\mathrm{d}}$ intra $/\left|J_{\text {inter }}\right|$ corresponding to Case $\mathrm{b}$, only two of the six states, the singlet ground state S0 and first excited triplet state T1, were stabilized far more than the other four states. The lowest two states dominated the thermal population, resulting in the effective two-spin system. The energy gap, $\Delta E^{\mathrm{ST}}$ eff, between the lowest singlet and triplet states corresponded to $\left|2 J_{\text {eff }}\right|$. The energy gap,
$\Delta E^{\mathrm{ST}}$ eff, in the range of Case $\mathrm{b},+10 \mathrm{~K} \leq \mathrm{J}^{\mathrm{d}}$ intra $/ k_{\mathrm{B}} \leq+100 \mathrm{~K}$ was in the order of $\Delta E^{\mathrm{ST}}$ eff $/ k_{\mathrm{B}} \sim 10^{1} \mathrm{~K}$. The two-spin system with $\left|2 \mathrm{~J}_{\text {eff }}\right| /$ $k_{\mathrm{B}} \sim 10^{1} \mathrm{~K} \gg\left|A^{\mathrm{d}}\right| / k_{\mathrm{B}}$ surely resulted in the nine-line spectra with half the spacing of $A / 2 g \mu_{\mathrm{B}} \sim 0.37 \mathrm{mT}$, as shown in the simulations based on equation (5) (Figure $\mathrm{S} 7$ in SI). It was concluded that $\pi$ dimers (DTP-P-NN ${ }^{*} \mathbf{2}^{2+}$ and $\left(\mathbf{E}_{2} \mathbf{P}_{2}-\mathbf{N N}^{*}\right) \mathbf{2}^{2+}$ had effective exchange interactions of $\left|2 J_{\text {eff }}\right| / k_{B}=\sim+10^{1} \mathrm{~K}$ through the $\pi$-cation dimer between the NN moieties based on equation (5) and $J_{\text {inter }} / k_{\mathrm{B}}=-$ $500 \mathrm{~K}$. In the case of $100 \mathrm{~K} \leq J^{\mathrm{d}}$ intra $/ k_{\mathrm{B}} \leq 400 \mathrm{~K}$ based on the $J_{\text {inter }} / k_{\mathrm{B}}$ values calculated using M06- $2 \mathrm{X},\left|2 J_{\text {eff }}\right| / k_{\mathrm{B}}$ become $1 \times 10^{1}-6 \times 10^{1} \mathrm{~K}$.

\section{Quantitative ESR}

To further characterize $J^{\mathrm{m}_{\text {intra, }},} J^{\mathrm{d}}$ intra, and $J_{\text {inter, }}$, the temperature dependence of paramagnetic susceptibility $\chi$ was examined in the temperature range of $100-160 \mathrm{~K}$ (frozen solid) and 190-280 K (solution) in DCM (melting point: $176 \mathrm{~K}$ ). The paramagnetic susceptibility $\chi$ was assumed to be proportional to double integrals D/s of ESR spectra. We chose quantitative ESR ${ }^{[69]}$ instead of SQUID magnetometry because the ratio of the biradical cations and residual NN unit (see above) needed to be checked soon after the reaction of the neutral precursor with $\mathrm{AgSbF}_{6}$ in $\mathrm{DCM}$ solution at room temperature. Once the ratio was confirmed, the solution was cooled down to $100 \mathrm{~K}$ to minimize decomposition of the biradical cations and then warmed to each data point. The product $\chi T$ of paramagnetic susceptibility and temperature was estimated with a nitroxide (TEMPOL) standard in DCM solution and its frozen solid by using equation (6),

$\chi T=\frac{\left[\text { standard] } \cdot D I_{\text {products }}\right.}{[\text { precursor }] \cdot D I_{\text {standard }}} \cdot N g^{2} \mu_{B}^{2} / 3 k_{B} S(S+1)_{\text {standard }}$

where $D /$ represents doubly integrated intensity in the whole range of scanned magnetic field and $N g^{2} \mu_{\mathrm{B}}^{2} / 3 k_{\mathrm{B}} S(S+1)$ term is $0.375 \mathrm{emu} \mathrm{mol}^{-1} \mathrm{~K}$ for the monoradical TEMPOL standard. Multiple independent measurements (4 times for biradical cations and 3 times for TEMOL) were conducted with a same cell to determine the error range.

Figure 8 shows the result of (DTP-P) ${ }^{++} \mathbf{N N}^{*}$ in DCM $(0.70 \mathrm{mM}$ (precursor)). Although the error range was relatively large partly due to the high relative permittivity of DCM, we could determine the boundary for each $\mathrm{J}$ values from the experiments. The analysis of ESR spectra recorded at $200 \mathrm{~K}$, i.e., just above the melting point of DCM, (Figure 7) has indicated that the $\pi$-dimer $(\text { DTP-P-NN })_{2}{ }^{2+}$ was considered to be principally present with a

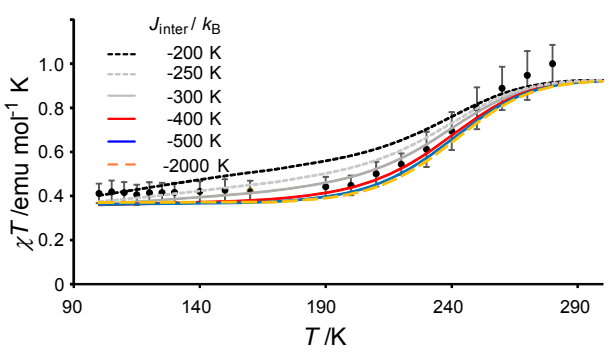

Figure 8. $\chi^{T-T}$ plot determined with quantitative ESR spectroscopy of (DTP$\mathbf{P})^{*+}-\mathrm{NN}^{*}$ in $\mathrm{DCM}(0.70 \mathrm{mM}$ (precursor) $)$ and theoretical curves $\left(\mathrm{J}_{\text {inter }} / \mathrm{K}_{\mathrm{B}}\right.$ : -200 , $250,-300,-400,-500,-2000 \mathrm{~K} ; J^{\mathrm{d}} \mathrm{intra}:+100 \mathrm{~K} ; J^{\mathrm{m}}$ intra: $+300 \mathrm{~K} ; \Delta H_{\mathrm{dim}}:-11 \mathrm{kcal}$ $\left.\mathrm{mol}^{-1}: \Delta S_{\text {dim }}-31 \mathrm{cal} \mathrm{mol}^{-1} \mathrm{~K}^{-1}\right)$. 
small amount of residual NN (4\%). The equilibrium is approximately shifted to full dimerization at $200 \mathrm{~K}$. At the lower temperatures, the $\pi$-dimer has dominant contribution to magnetic susceptibility. The susceptibility of the dimer has been derived from the spin Hamiltonian composed of the first and second terms of equation (4) and can be written as equation (7), ${ }^{[57,68]}$

$\chi(\pi$-dimer $)=\frac{N_{\mathrm{d}} g_{\mathrm{d}}^{2} \mu_{\mathrm{B}}^{2}}{3 k_{\mathrm{B}} T} \frac{\sum_{i} S_{i}\left(S_{i}+1\right)\left(2 S_{i}+1\right) \exp \left(-E\left(S_{i}\right) / k_{\mathrm{B}} T\right)}{\sum_{i}\left(2 S_{i}+1\right) \exp \left(-E\left(S_{i}\right) / k_{\mathrm{B}} T\right)}$

where the value of resultant spin $S_{i}$ are 2, 1, and 0 for quintet, triplet, and singlet states, respectively, and $E\left(S_{\mathrm{i}}\right)$ denotes eigenenergy for each eigenstate (See SI for details). Below 160 $\mathrm{K}$, the $\chi^{T}$ values were nearly constant at ca. $0.4 \mathrm{emu} \mathrm{K} \mathrm{mol}{ }^{-1}$. If the interaction ratio $J_{\text {inter }} / \mid J^{\mathrm{d}}$ intra $\mid$ in the $\pi$-dimer falls within those of Case $b$, the thermal population of the four-spin system is dominated by the lowest singlet S0 and the first excited triplet T1 states with $\Delta E^{\mathrm{ST}}{ }_{\text {eff }}=E_{\mathrm{T} 1}-E_{\mathrm{SO}_{0}}^{[57,68]}$ as stated above, in an intermediate temperature range of $\Delta E^{\mathrm{ST}}$ eff $<<k_{\mathrm{B}} T<<E_{\mathrm{Q} 2}-E_{\mathrm{T} 1}\left(E_{\mathrm{Q} 2}\right.$ is the second excited state: see Figure $\mathrm{S} 8$ in SI for the energy spectrum of the four-spin system). The quasi-degeneracy of the low-lying S0 and T1 states gives the effective two-spin system, which behaves as two apparently decoupled $S=1 / 2$ spins, ${ }^{[57,68]}$ giving $\chi T=0.375 \mathrm{emu} \mathrm{K} \mathrm{mol}^{-1}$ with a horizontally flat plot of $\chi T$ vs. $T$. From the simulation based on equation 7 , we found that the intermolecular interaction should have the boundary of $J_{\text {inter }} / k_{\mathrm{B}} \leq-$ $300 \mathrm{~K}$ in the case of $\mathrm{J}^{\mathrm{d}}{ }_{\text {intra }} / k_{\mathrm{B}}=+100 \mathrm{~K}$ (Figure 8), which were consistent with the results of the above simulations of the ESR signal in solution. In addition, it was also found that the upper limit of $J \mathrm{~d}_{\text {intra }} / k_{\mathrm{B}}$ was $+100 \mathrm{~K}$ and $+200 \mathrm{~K}$ in the cases of $J_{\text {inter }}=-500 \mathrm{~K}$ (ESR simulation) and $-1719 \mathrm{~K}(\mathrm{M} 06-2 \mathrm{x})$, respectively (see Figure S9 in SI).

When the temperature is raised, higher excited states for the $\pi$ dimer would recover the thermal population. In the hightemperature range above $190 \mathrm{~K}$, the dissociated monomer biradical cation with triplet ground state also gradually contributes to total $\chi^{T}$ values in solution. The temperature variation of susceptibility $\chi$ of the dimer and a shift of dimmerization equilibrium with changing the molar ratio of [ $\pi$-dimer] : [biradical cation] should occur simultaneously. Thus, the doubly integrated intensity $D I$ was assumed to be proportional to magnetic susceptibility $\chi$ for all of the paramagnetic species in solution as shown in equations (8) and (9),

$D I($ obs $)=D I(\pi$-dimer $)+D I($ biradical cation $)+D I($ residual $\mathrm{NN})$ (8)

$D I(\pi$-dimer $): D I($ biradical cation $): D I($ residual $\mathrm{NN})=$

[ $\pi$-dimer] $\chi(\pi$-dimer $):$ [biradical cation] $\chi$ (biradical cation $):[$ residual $\mathrm{NN}]$ $\chi($ residual $\mathrm{NN})$

where the paramagnetic susceptibilities $\chi$ were expressed in equations (2) and (7). The molar ratio of [ $\pi$-dimer] : [biradical cation] was assumed to be governed by the dimerization equilibrium constant $K_{\text {dim }}$, while the concentration [residual $\mathrm{NN}$ ] was independent of temperature. The temperature variation of $K_{\text {dim }}$ was estimated using the dimerization enthalpy and entropy $\Delta H_{\text {dim }}=-11 \mathrm{kcal} \mathrm{mol}^{-1}$ and $\Delta S_{\text {dim }}=-31 \mathrm{cal} \mathrm{mol}^{-1} \mathrm{~K}^{-1}$. These values reproduced the temperature variation of the absorbance at 815 $\mathrm{nm}$ in the VT-UV-vis-NIR measurements (See Figures 6(a) and $\mathrm{S} 10 \mathrm{in} \mathrm{SI}$ ) and were in the similar range for those of $\pi$-dimerization of other quaterthiophene radical cations. ${ }^{[35,38]}$ In this case, the increase of total $\chi T$ with increasing temperature was found to be dominantly caused by the contribution of monomer biradical cation as shown in Figure $\mathrm{S} 11$ in SI. Setting the exchange interactions in the dimer $J_{\text {inter }} / k_{\mathrm{B}}=-500 \mathrm{~K}$ and $J_{\text {intra }}^{\mathrm{d}} / \mathrm{k}_{\mathrm{B}}=100 \mathrm{~K}$ (ESR simulation), it was found that the lower limit of $J^{m_{i n t r a}}$ in the dissociated monomer (DTP-P) ${ }^{++}-N^{*}$ was $\mathrm{J}_{\text {intra }} \geq 300 \mathrm{~K}$ (see Figure S12 in SI). Thus, the result of B97D3 $(+5 \mathrm{~K})$ was found to be apparently small, and it was indicated that this method was inappropriate for the predication of $\mathrm{Jm}_{\text {intra. }}$.

The $\chi^{T-T}$ plot was also obtained for $\left(\mathrm{E}_{2} \mathrm{P}_{2}\right)^{-+}-\mathrm{NN}^{*}$ in DCM $(0.25$ $\mathrm{mM}$, (Figure $\mathrm{S} 13$ in $\mathrm{SI}))$. $\left(\mathbf{E}_{2} \mathbf{P}_{2}-\mathbf{N N}\right)_{2}{ }^{2+}$ in the temperature range of $100-160 \mathrm{~K}$ gave a similarly flat plot with the averaged $\chi T$ value of near 0.375 , but the error range was much larger than that of (DTP-P) ${ }^{-+}-\mathbf{N N}^{\cdot}$ due to the instability of $\left(\mathrm{E}_{2} \mathbf{P}_{2}\right)^{-+}-\mathbf{N N}^{*}$. In the temperature range above $190 \mathrm{~K}$, the radical cation moiety of $\left(E_{2} P_{2}\right)^{-+}-N^{*}$ was gradually decomposed and the ratio of residual $\mathrm{NN}$ to $\left(\mathrm{E}_{2} \mathbf{P}_{2}\right)^{-+}-\mathbf{N N}^{*}$ increased. The $\chi T$ value reached only to 0.7 at $280 \mathrm{~K}$ because of the decomposition. Therefore, it was difficult to

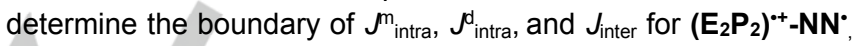
but it would be comparable to those of (DTP-P) ${ }^{+*}-\mathbf{N N}^{*}$ considering the results of simulations of ESR in solution and DFT calculations.

\section{DFT calculations of $\pi$-dimers}

To further gain insight into the electronic structures of (DTP-P$\left.\mathrm{NN}^{*}\right)_{2}{ }^{2+}$ and $\left(\mathrm{E}_{2} \mathrm{P}_{2}-\mathbf{N N}\right)_{2}{ }_{2}{ }^{2+}$, DFT calculations were conducted. For the estimation of $J_{\text {inter, }}$, the $\pi$-dimers without NN moieties, $(5)_{2}{ }^{2+}$ and $(8) 2^{2+}$, were also calculated. Thus, geometry optimizations of (DTP-P-NN $)_{2}{ }^{2+},\left(\mathrm{E}_{2} \mathrm{P}_{2}-\mathrm{NN}^{*}\right)_{2}{ }^{2+},(5)_{2}{ }^{2+}$, and $(8)_{2}{ }^{2+}$ at both the singlet and triplet states were performed using the B97D3(BJ) and M06$2 X$ methods with the 6-31G(d) basis set. The optimized structures are shown in Figure 9 (for BS-singlet of (DTP-P-NN $)_{2}{ }^{2+}$ and $\left(\mathbf{E}_{2} \mathbf{P}_{2-}\right.$ $\left.\mathrm{NN}^{*}\right)_{2}{ }^{2+}$ at the M06-2X/6-31G(d) level) and Figure S14 in SI (for other structures), and the numerical results are summarized in Table 2.

(a)
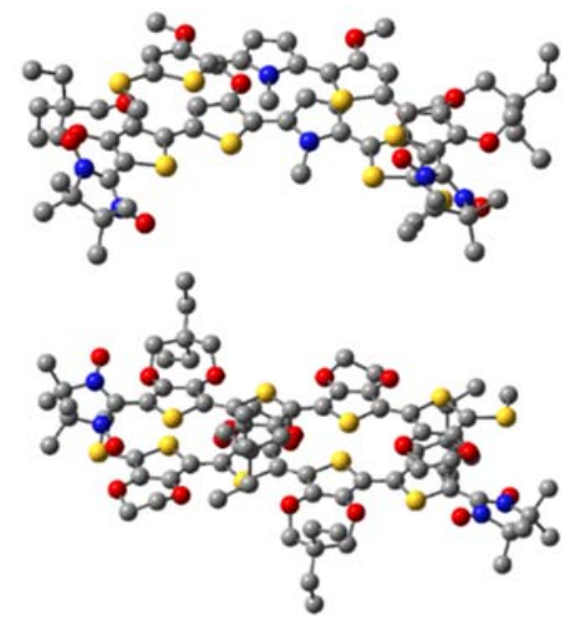

Figure 9. Optimized structures at BS singlet states of (a) (DTP-P-NN $)_{2}{ }^{2+}$ and (b) $\left(\mathrm{E}_{2} \mathrm{P}_{2}-\mathrm{NN}^{*}\right)_{2}{ }^{2+}$ at the M06-2X/6-31G(d) level. Hydrogen atoms are omitted. 
Table 2. Total energies $E$ and $\left\langle S^{2}\right\rangle$ values (with shortest intermolecular C-C distance $d_{c-c}$ ) for the BS-singlet and high spin (Triplet) states and calculated exchange interactions at $\pi$-dimer moiety $J_{\text {inter }}$ and overall units $J_{\text {oa(ST) }}$ of $(5)_{2}{ }^{2+},(8) 2_{2}{ }^{2+},(\text { DTP-P-NN })_{2}{ }^{2+}$, and $\left.\left(E_{2} \mathbf{P}_{2}-N^{-}\right)^{*}\right)_{2}{ }^{2+}$

\begin{tabular}{|c|c|c|c|c|c|c|c|}
\hline compd & Method $^{[a]}$ & $E_{\mathrm{BS}} /$ hartree & $E_{\mathrm{HS}} /$ hartree & $\left\langle\mathrm{S}^{2}\right\rangle_{\mathrm{BS}}\left(d_{\mathrm{C}-\mathrm{C}} / \AA\right)$ & $\left\langle S^{2}\right\rangle_{\mathrm{HS}}\left(d_{\mathrm{C}-\mathrm{C}} / \AA\right)$ & $J_{\text {inter }} k_{\mathrm{B}}{ }^{-1 / K}$ & $J_{\mathrm{oa}(\mathrm{ST})} k_{\mathrm{B}}^{-1 / K}$ \\
\hline \multirow[t]{2}{*}{$(5)_{2}{ }^{2+}$} & B97D3(BJ) & -5989.64456 & -5989.62661 & $0.0000(3.052)^{[b]}$ & $2.0090(3.351)^{[b]}$ & -2822 & - \\
\hline & M06-2X & -5989.88411 & -5989.87320 & $0.0000(2.951)^{[b]}$ & $2.0055(3.143)^{[\mathrm{b}]}$ & -1719 & - \\
\hline \multirow[t]{2}{*}{$(8) 2^{2+}$} & B97D3(BJ) & -7898.16885 & -7898.163548 & $0.0000(3.348)^{[c]}$ & $2.0095(3.469)^{[c]}$ & -833 & - \\
\hline & M06-2X & -7898.58100 & -7898.57668 & $0.9528(3.350)^{[c]}$ & $2.0881(3.4135)^{[c]}$ & -1201 & - \\
\hline \multirow[t]{2}{*}{$\left(\right.$ DTP-P-NN') ${ }^{2+}$} & B97D3(BJ) & -7055.47802 & $-7055.47807^{d}$ & $1.0464(3.095)^{[b]}$ & $2.0407(3.095)^{[\mathrm{b}, \mathrm{d}]}$ & - & +16 \\
\hline & M06-2X & -7055.87649 & $-7055.87521^{d}$ & $1.7061(3.059)^{[b]}$ & $2.5208(2.969)^{[\mathrm{b}, \mathrm{d}]}$ & & -499 \\
\hline \multirow[t]{2}{*}{$\left(E_{2} P_{2}-N^{*}\right)_{2}{ }^{2+}$} & B97D3(BJ) & -8964.02197 & $-8964.02190^{d}$ & $1.0812(3.441)^{[c]}$ & $2.0312(3.436)^{[c, d]}$ & & -23 \\
\hline & M06-2X & -8964.59207 & $-8964.59084^{d}$ & $2.2958(3.292)^{[\mathrm{e}]}$ & $3.2401(3.266)^{[\mathrm{d}, \mathrm{e}]}$ & - & -411 \\
\hline
\end{tabular}

[a] Using the 6-31G(d) basis set. [b] Between a pyrrole $\beta$-carbon and thiophene $\beta$-carbon. [c] Between thiophene $\alpha$-carbons. [d] BS method was applied. [e] Between thiophene $\alpha$ - and $\beta$-carbons.

Concerning the structures of (DTP-P-NN $)_{2}{ }^{2+}$ and $\left(\mathrm{E}_{2} \mathrm{P}_{2}-\mathrm{NN}^{*}\right)_{2}{ }^{2+}$, the "head-to-tail" dimers (Figures 9 and $\mathrm{S} 14$ in $\mathrm{SI}$ ) were more stable, while no "head-to-head" dimer with comparable total energy could be optimized due to the steric bulkiness of the NN units. The BS-triplet states also had structures quite similar to those of the BS-singlet states (Table 2).

For radical cations of oligothiophenes, larger spin densities were found on $\mathrm{sp}^{2}$ carbons rather than sulfur atoms. Therefore, the SOMO-SOMO interaction resulted in short $\mathrm{C}-\mathrm{C}$ contact, typically less than the sum of the van der Waals radius (3.4 $\AA$ ), with a slipped stack structure to avoid repulsion between $S$ atoms. ${ }^{[35]}$ On the other hand, the $\mathrm{N}-\mathrm{C}-\mathrm{C}-\mathrm{S}$ dihedral angle involving the formal $\mathrm{C}=\mathrm{N}$ bond in the $\mathrm{NN}$ unit and the $\mathrm{C}-\mathrm{S}$ bond in ProDOT unit should affect exchange interaction between the $\mathrm{NN}$ radical and radical cation moieties. ${ }^{[63]}$ However, changes in the dihedral angles before and after dimerization were negligibly small for both (DTP$\left.\mathbf{P}-\mathrm{NN}^{\bullet}\right)_{2}{ }^{2+}$ and $\left(\mathrm{E}_{2} \mathrm{P}_{2}-\mathrm{NN}^{\cdot}\right)_{2}{ }^{2+}$. The optimized structures of $(\mathbf{5})_{2}{ }^{2+}$ and (8) $2^{2+}$ at both (BS-)singlet and triplet states were similar to those of the corresponding oligomer moieties in (DTP-P-NN $)_{2}{ }^{2+}$ and $\left(E_{2} P_{2}-N^{*}\right)_{2}{ }^{2+}$, respectively. For the "pure" DFT of B97D3, the $\left\langle S^{2}\right\rangle_{\mathrm{BS}}$ values of both $(5)_{2}{ }^{2+}$ and $(8)_{2}{ }^{2+}$ at singlet states were 0.0000 , indicating the closed shell nature of the $\pi$-dimers at this level of theory. The $\left\langle S^{2}\right\rangle_{\mathrm{BS}}$ value of $(\mathbf{5})_{2}{ }^{2+}$ was also 0.0000 , even using the hybrid DFT of the M06-2X method. In contrast, the presence of some diradical characters in the M06-2X structure of $(8) 2^{2+}$ was shown by the $\left\langle S^{2}\right\rangle_{B S}$ value of 0.9528 and NOON diradical index of 0.652 . In all cases, the radical character of the oligomer moieties was significantly reduced upon $\pi$-dimerization. Despite the differences in diradical character between the B97D3 and M06-2X structures of (8) $\mathbf{2}^{2+}$, the calculated $J_{\text {inter }}$ was around $1000 \mathrm{~K}$, while $(5)_{2}{ }^{2+}$ was predicted to have a larger exchange interaction of around -2000 to $-3000 \mathrm{~K}$. These theoretical results are consistent with the experimental analysis $\left(\mathrm{J}_{\text {inter }} / K_{\mathrm{B}} \leq-500 \mathrm{~K}\right)$ for (DTP-P-NN $)_{2}{ }^{2+}$ and $\left(\mathrm{E}_{2} \mathbf{P}_{\mathbf{2}}-\mathbf{N N}\right)_{2}{ }_{2}{ }^{2+}$. Similar antiferromagnetic exchange interactions were also shown in phenalenyl $\pi$-dimers $\left(J / k_{B}=-1000 \mathrm{~K}\right)$, in which the radical centers were on carbon atoms. ${ }^{[70]}$

The B97D3 calculations for both (DTP-P-NN $)_{2}{ }^{2+}$ and $\left(\mathbf{E}_{2} \mathbf{P}_{\mathbf{2}^{-}}\right.$ $\left.\mathbf{N N}^{*}\right)_{2}{ }^{2+}$ gave $\left\langle\mathrm{S}^{2}\right\rangle_{\mathrm{BS}}$ and $\left\langle\mathrm{S}^{2}\right\rangle_{\mathrm{HS}}$ values of around 1 and 2 , respectively. Given that the ideal $\left\langle S^{2}\right\rangle$ values for the BS-singlet with $100 \%$ diradical character and triplet were 1 and 2 , respectively, these results suggested that the $\pi$-dimer moieties were of near-closed-shell nature at this level of theory, as were $(5) 2^{2+}$ and $(8) 2^{2+}$. In fact, the spin densities calculated with the
B97D3 method (Figure S15 in SI) mainly resided on the NN units, with a small spin density found in the oligomer moieties. Spin flipping from singlet to triplet states was observed in the $\mathrm{NN}$ units, whereas the direction of the total spin density within the intramolecular NN and oligomer moieties remained parallel for both singlet and triplet states (see arrow directions in Figure S15). Thus, the small spin distributions in the oligomer moieties were considered to be caused by delocalization from the $\mathrm{NN}$ radical into the closed-shell $\pi$-dimer dication. Therefore, the overall exchange interaction ( $\left.\mathrm{J}_{\mathrm{Oa}(\mathrm{ST})}\right)$ of (DTP-P-NN $)_{2}{ }^{2+}$ and $\left(\mathrm{E}_{2} \mathbf{P}_{2}-\mathrm{NN}^{*}\right)_{2}{ }^{2+}$ calculated with the BS-singlet and BS-triplet states of B97D3 structures based on equation (3) mainly involved the character of the longdistance spin communication between NN units through the closed-shell $\pi$-dimers. As a result, the $J_{\text {oa(ST) }}$ values of (DTP-P$\left.\mathbf{N N}^{*}\right)_{2}{ }^{2+}(+16 \mathrm{~K})$ and $\left(\mathrm{E}_{2} \mathbf{P}_{2}-\mathbf{N N}^{*}\right) \mathbf{2}^{2+}(-23 \mathrm{~K})$ from the B97D3 method were calculated to be relatively small. However, considering that the pure DFT overstabilized the closed-shell singlet state as discussed in the section of DFT calculations of oxidized monomers, the calculated electronic structure would not depict the actual electronic structure.

The $\left\langle S^{2}\right\rangle_{\text {BS }}$ and $\left\langle S^{2}\right\rangle_{\text {HS }}$ values for (DTP-P-NN $)_{2}{ }^{2+}$ and $\left(E_{2} P_{2-}\right.$ $\left.\mathrm{NN}^{*}\right)_{2}{ }^{2+}$ from the hybrid DFT of M06-2X were larger than those from the pure DFT of B97D3 (see Table 2), because some diradical character was expected to be present in the $\pi$-dimer moieties. The spin distributions from the M06-2X method are shown in Figure 10. As predicted, substantial spin densities, albeit less than 1, were present in the oligomer moieties at this level of theory. The stronger SOMO-SOMO interaction in the $\pi$-dimer moiety of (DTP-P-NN $)^{*}{ }^{2+}$ caused smaller diradical character in the oligomer moiety, resulting in smaller spin densities (0.41$0.56)$ than those of $\left(E_{2} P_{2}-N_{N} \cdot\right)_{2}{ }^{2+}(0.87-0.98)$. Spin flipping from singlet to triplet was also observed in the NN units (see arrow directions in Figure 10), as shown in the above B97D3 structures in Figure S15. However, the directions of the total spin densities within the intramolecular NN and oligomer moieties in the bottom $\mathrm{NN}$-oligomer molecules in both BS-triplet states (Figures $10 \mathrm{~b}$ and 10d) were flipped, probably because of the strong antiferromagnetic interaction at the $\pi$-dimer moieties. Thus, the $J_{\text {oa(ST) }}$ in the M06-2X structures mainly involved the character of the exchange interaction within the bottom monomer part, which would be closely related to $\mathrm{J}^{\mathrm{d}}$ intra, and the calculated values were $-499 \mathrm{~K}$ for (DTP-P-NN $)_{2}{ }^{2+}$ and $-411 \mathrm{~K}$ for $\left(\mathbf{E}_{2} \mathbf{P}_{2}-\mathbf{N N}^{*}\right)_{2}{ }^{2+}$. These values were smaller than the $\mathrm{Jm}_{\text {intra }}$ values in the monomer structures from the M06-2X method shown in Table 1 ((DTP-P) ${ }^{-+}$- 
$\left.\mathbf{N N}^{*},+918 \mathrm{~K} ;(\mathbf{E 2 P 2})^{*+}-\mathbf{N N}^{\bullet},+1350 \mathrm{~K}\right)$. The smaller $\mathrm{J}_{\text {oa(ST) }}$ would be caused by the smaller (di)radical character in the $\pi$-dimer moieties than in the monomer $\mathrm{NN}$-substituted radical cations, which reduced the intramolecular exchange interaction in the bottom units in Figures $10 \mathrm{~b}$ and $10 \mathrm{~d}$. However, the $\mathrm{J}_{\mathrm{oa}(\mathrm{ST})}$ for (DTP-P$\left.\mathbf{N N}^{*}\right)^{2+}(-499 \mathrm{~K})$ may be overestimated judging from the upper limit of $J_{\text {intra }}^{\mathrm{d}}\left(\leq 200 \mathrm{~K}\right.$ for $\left.J_{\text {inter }}=-1719 \mathrm{~K}\right)$ from the quantitative ESR experiment.
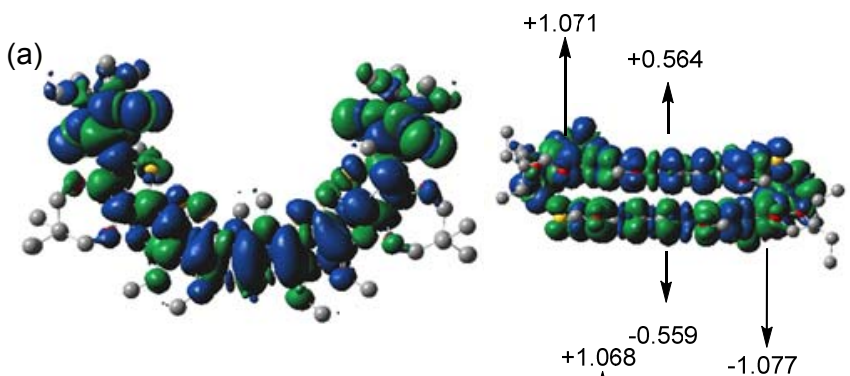

(b)
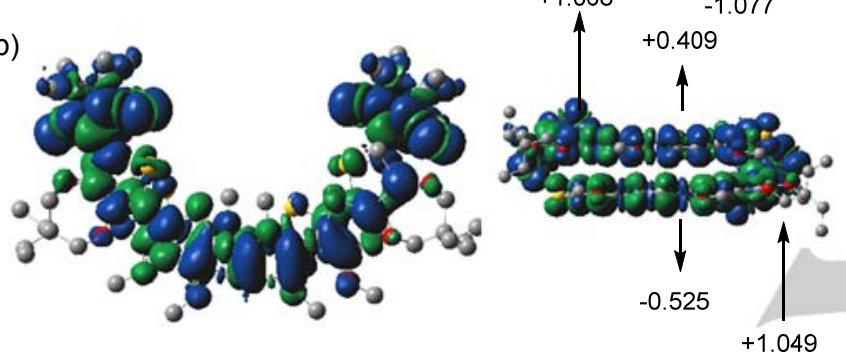

(c)
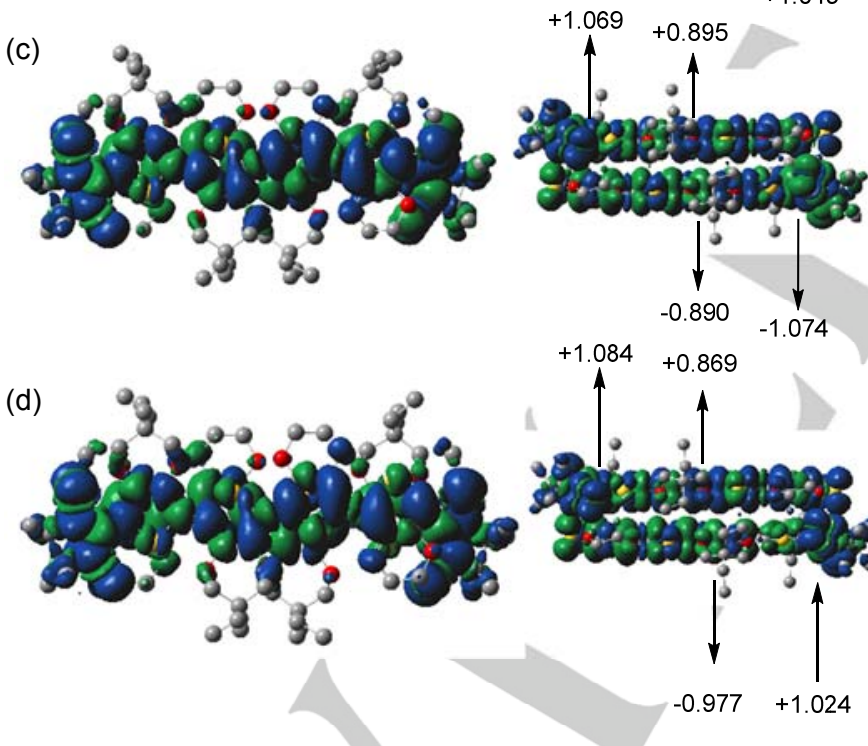

Figure 10. Spin distributions of (DTP-P-NN $)_{2}{ }^{2+}$ at (a) BS-singlet and (b) BStriplet states, and $\left(\mathrm{E}_{2} \mathrm{P}_{2}-\mathrm{NN}\right)_{2}{ }^{2+}$ at (c) BS-singlet and (d) BS-triplet states, calculated using the M06-2X method (isovalue $=0.0004$ ). Direction and length of the arrow denote the total spin densities of $\mathrm{NN}$ and oligomer moieties.

For the magnitude of exchange interactions between NN units through molecular wires, previous B3LYP calculations demonstrated that "vertical" $2 \mathrm{~J} / \mathrm{hc}$ values for neutral quaterthiophene ( $\left.\mathbf{N N}^{\bullet}-\mathbf{T}_{4}-\mathbf{N N}^{*}\right)$ was $-54.05 \mathrm{~cm}^{-1}\left(\mathrm{~J} / \mathrm{k}_{\mathrm{B}}=-38.6\right.$
$\mathrm{K}) .{ }^{[47]}$ The "adiabatic" $J / k_{\mathrm{B}}$ value using the same method was $39.6 \mathrm{~K}$. In the case of B97D3 and M06-2X, the "adiabatic" $\mathrm{J} / \mathrm{k}_{\mathrm{B}}$ values were $-17.5 \mathrm{~K}$ and $-15.4 \mathrm{~K}$, respectively. These exchange interactions were caused by similar spin polarization mechanisms (see Figure S16 in SI) as observed in the B97D3 structures (see Figure S15). The $J_{\text {oa(ST) }}$ values calculated from the B97D3 method for (DTP-P-NN $)_{2}{ }^{2+}(+16 \mathrm{~K})$ and $\left(\mathrm{E}_{2} \mathbf{P}_{2}-\mathbf{N N}^{*}\right)_{2}{ }^{2+}(-23 \mathrm{~K})$ were comparable to that of quaterthiophene at the same level of theory. In contrast, as a related example, a van der Waals dimer of neutral terthiophene $\left(\mathbf{T}_{4}-\mathbf{N N}^{*}\right)_{2}$ (see Figure $\mathrm{S} 17$ in $\mathrm{SI}$ for the optimized structures) was less efficient for electronic communication between the NN units through the dimer $(J=-9.1$ $\mathrm{K}$ with B97D3, $\mathrm{J}=-1.0 \mathrm{~K}$ with M06-2X). Therefore, the $\pi$-dimer structures between radical pairs played a vital role in the better electronic communication between $\mathrm{NN}$ biradicals through molecular wires. The much greater $J_{\mathrm{oa}(\mathrm{ST})}$ values, shown in the M06-2X structures of (DTP-P-NN $)_{2}{ }^{2+}(-499 \mathrm{~K})$ and $\left(\mathrm{E}_{2} \mathbf{P}_{\mathbf{2}}-\mathrm{NN}^{*}\right)_{2}{ }^{2+}$ $(-411 \mathrm{~K})$, were due to the effect of spin polarization within one monomer unit because of a substantial diradical character and fairly strong intermolecular exchange interaction $\left(J_{\text {inter }}\right)$ in the $\pi$ dimer moieties. Based on the results of higher theoretical calculations (RASSCF) of radical cation $\pi$-dimers, ${ }^{[64]}$ the presence of some diradical character in the radical $\pi$-dimer moieties can be considered, resulting in M06-2X giving better electronic structure predictions for (DTP-P-NN $)_{2}{ }^{2+}$ and $\left(\mathbf{E}_{2} \mathbf{P}_{2}-\mathbf{N N}^{*}\right)_{2}{ }^{2+}$, even though the $J_{\mathrm{oa}(\mathrm{ST})}$ values may be overestimated. In any case, comparable or larger spin exchange interactions between the $\mathrm{NN}$ biradicals in (DTP-P-NN $)_{2}{ }^{2+}$ and $\left(\mathrm{E}_{2} \mathrm{P}_{2}-\mathrm{NN}^{*}\right)_{2}{ }^{2+}$ indicated that these oligomer radical cation $\pi$-dimers acted as superior molecular wires for electronic communication.

\section{Conclusions}

In the present study, radical cation $\pi$-dimers of conjugated oligomers, in which two NN radical moieties were arranged at diagonal positions of the oligomer ends, were successfully designed and prepared. ESR spectra of these systems in the solution phase showed that long-distance spin communication between the NN units operated through radical cation $\pi$-dimers. DFT calculations showed that the magnitude of the exchange interactions was comparable or larger than that of covalently linked neutral quaterthiophene. Therefore, the radical cation $\pi$ dimers of the conjugated oligomers were demonstrated to behave as efficient molecular wires for electronic communication for the first time. Considering the intimate relationship between electronic communication and electronic conduction through molecular wires, the conjugated oligomer radical cation $\pi$-dimers are expected to be a component of redox-responsible conductive supramolecular systems. For example, a new switching device based on the molecular wire properties of $\pi$-dimers may be developed from gold nanoparticles covered with the conjugated oligomers. ${ }^{[71]}$ In addition to the expected conductive properties, the strong exchange interactions in the $\pi$-dimer moiety may be applicable to spintronics. 


\section{Acknowledgements}

This work was supported by a Grant-in-Aid for Scientific Research on Innovative Areas (15H00954).

Keywords: Radical ions, Radicals, Exchange interactions, Electronic structure, Density functional calculations

[1] D. M. Guldi, H. Nishihara, L. Venkataraman, Chem. Soc. Rev. 2015, $44,842-844$.

[2] L. D. A. Siebbeles, F. C. Grozema, Eds., Charge and Exciton Transport through Molecular Wires, Wiley, Weinheim, 2011.

[3] L. de Cola, Ed., Molecular Wires, Springer, Berlin, 2005

[4] N. J. Tao, Nat. Nanotecnol. 2006, 1, 173-181.

[5] C. Joachim, M. A. Ratner, Proc. Natl. Acad. Sci. USA. 2005, 102 8801-8808.

[6] C. Lambert, in Organic Redox Systems (Ed.: T. Nishinaga), Wiley, Hoboken, 2016, pp. 245-268.

[7] C. Schubert, J. T. Margraf, T. Clark, D. M. Guldi, Chem. Soc. Rev. 2015, 44, 988-998.

[8] T. Tanaka, A. Osuka, Chem. Soc. Rev. 2015, 44, 943-969.

[9] J. P. Launay, Coord. Chem. Rev. 2013, 257, 1544-1554.

[10] O. S. Wenger, Chem. Soc. Rev. 2012, 41, 3772-3779.

[11] I. Ratera, J. Veciana, Chem. Soc. Rev. 2012, 41, 303-349.

[12] J. M. Tour, Acc. Chem. Res. 2000, 33, 791-804.

[13] S. Vela, S. Bauroth, C. Atienza, A. Molina-Ontoria, D. M. Guldi, N Martín, Angew. Chemie Int. Ed. 2016, 55, 15076-15080.

[14] F. Wessendorf, B. Grimm, D. M. Guldi, A. Hirsch, J. Am. Chem. Soc. 2010, 132, 10786-10795.

[15] J. L. Sessler, B. Wang, A. Harriman, J. Am. Chem. Soc. 1993, 115 10418-10419.

[16] Y. Tanaka, M. Kiguchi, M. Akita, Chem. Eur. J. 2017, 23, 4741-4749.

[17] C.-Y. Yeh, C.-C. Wang, C.-H. Chen, S.-M. Peng, in Redox Systems under Nano-Space. Control (Ed.: T. Hirao), Springer, Berlin, 2006, pp. 85-117.

[18] M. D. Ward, Chem. Soc. Rev. 1995, 24, 121-134.

[19] R. Sakamoto, K.-H. Wu, R. Matsuoka, H. Maeda, H. Nishihara, Chem. Soc. Rev. 2015, 44, 7698-7714.

[20] A. Harriman, R. Ziessel, Chem. Commun. 1996, 1707-1716.

[21] K. E. Preuss, Polyhedron 2014, 79, 1-15.

[22] T. Nishinaga, K. Komatsu, Org. Biomol. Chem. 2005, 3, 561-569.

[23] C. Kahlfuss, E. Saint-Aman, C. Bucher, in Organic Redox Systems (Ed.: T. Nishinaga), Wiley, Hoboken, 2016, pp. 39-88.

[24] D. W. Zhang, J. Tian, L. Chen, L. Zhang, Z. T. Li, Chem. Asian J. 2015 , 10, 56-68.

[25] T. Nishinaga, in Organic Redox Systems (Ed.: T. Nishinaga), John Wiley, Hoboken, 2016, pp. 383-410.

[26] L. L. Miller, K. R. Mann, Acc. Chem. Res. 1996, 29, 417-423.

[27] M. G. Hill, K. R. Mann, L. L. Miller, J.-F. Penneau, J. Am. Chem. Soc. 1992, 114, 2728-2730.

[28] P. Bäuerle, U. Segelbacher, A. Maier, M. Mehring, J. Am. Chem. Soc 1993, 115, 10217-10223.

[29] Y. Yu, E. Gunic, B. Zinger, L. L. Miller, J. Am. Chem. Soc. 1996, 118, 1013-1018.

[30] E. Levillain, J. Roncali, J. Am. Chem. Soc. 1999, 121, 8760-8765.

[31] J. J. Apperloo, R. A. J. Janssen, P. R. L. Malenfant, L. Groenendaal, J. M. J. Fréchet, J. Am. Chem. Soc. 2000, 122, 7042-7051.

[32] T. Sakai, T. Satou, T. Kaikawa, K. Takimiya, T. Otsubo, Y. Aso, J. Am. Chem. Soc. 2005, 127, 8082-8089.

[33] J. Casado, K. Takimiya, T. Otsubo, F. J. Ramírez, J. J. Quirante, R. Ponce Ortiz, S. R. González, M. Moreno Oliva, J. T. López Navarrete, J. Am. Chem. Soc. 2008, 130, 14028-140289.

[34] S. Klod, K. Haubner, E. Jähne, L. Dunsch, Chem. Sci. 2010, 1, 743750 .

[35] M. Tateno, M. Takase, M. Iyoda, K. Komatsu, T. Nishinaga, Chem. Eur. J. 2013, 19, 5457-5467.

[36] Y. le, Y. Okamoto, S. Tone, Y. Aso, Chem. Eur. J. 2015, 21, 1668816695.

[37] M. Hasegawa, K. Kobayakawa, H. Matsuzawa, T. Nishinaga, T. Hirose, K. Sako, Y. Mazaki, Chem. Eur. J. 2017, 23, 3267-3271.
[38] T. Nishinaga, Y. Sotome, J. Org. Chem. 2017, 82, 7245-7253.

[39] D. D. Graf, R. G. Duan, J. P. Campbell, L. L. Miller, K. R. Mann, J. Am. Chem. Soc. 1997, 119, 5888-5899.

[40] D. Yamazaki, T. Nishinaga, N. Tanino, K. Komatsu, J. Am. Chem. Soc. 2006, 128, 14470-14471.

[41] X. Chen, B. Ma, S. Chen, Y. Li, W. Huang, J. Ma, X. Wang, Chem. Asian J. 2013, 8, 238-243.

[42] E. F. Ullman, J. H. Osiecki, D. G. B. Boocock, R. Darcy, J. Am. Chem. Soc. 1972, 94, 7049-7059.

[43] K. Higashiguchi, K. Yumoto, K. Matsuda, Org. Lett. 2010, 12, 52845286.

[44] M. Shinomiya, K. Higashiguchi, K. Matsuda, J. Org. Chem. 2013, 78, 9282-9290.

[45] M. L. Kirk, D. a Shultz, D. E. Stasiw, F. Lewis, G. Wang, C. L. Brannen, R. D. Sommer, P. D. Boyle, J. Am. Chem. Soc. 2013, 135, 1714417154.

[46] C. Herrmann, J. Elmisz, Chem. Commun. 2013, 49, 10456-10458.

[47] S. Nishizawa, J. Y. Hasegawa, K. Matsuda, J. Phys. Chem. C 2015, 119, 5117-5121.

[48] Y. Tsuji, R. Movassagh, S. Datta, R. Hoffmann, ACS Nano 2015, 9 $11109-11120$

[49] K. Matsuda, M. Irie, J. Am. Chem. Soc. 2000, 122, 8309-8310.

[50] K. Matsuda, M. Irie, J. Am. Chem. Soc. 2000, 122, 7195-7201.

[51] K. Matsuda, M. Irie, Chem. Eur. J. 2001, 7, 3466-3473.

[52] T. Nishinaga, T. Kageyama, M. Koizumi, K. Ando, M. Takase, M. Iyoda, J. Org. Chem. 2013, 78, 9205-9213.

[53] S. Suzuki, K. Okada, in Organic Redox Systems (Ed.: T. Nishinaga), Wiley, Hoboken, 2016, pp. 269-285.

[54] R. Tanimoto, S. Suzuki, M. Kozaki, K. Okada, Chem. Lett. 2014, 43, 678-680.

[55] C. Lin, T. Endo, M. Takase, M. lyoda, T. Nishinaga, J. Am. Chem. Soc. 2011, 133, 11339-11350.

[56] T. Nishinaga, M. Tateno, M. Fujii, W. Fujita, M. Takase, M. lyoda, Org. Lett. 2010, 12, 5374-5377.

[57] Y. Kanzaki, D. Shiomi, K. Sato, T. Takui, J. Phys. Chem. B 2012, 116, 1053-1059.

[58] The spectral simulations were made by use of the program package "EasySpin": S. Stoll, A. Schweiger, J. Magn. Reson. 2006, 178, 42-55. The solution-phase ESR spectra were simulated by applying the function for solid-state spectra in "EasySpin", which includes exchange interaction terms represented by scalar products of spin operators. It was assumed that both $g$ and $A$ tensors were isotropic with single principal values, which gave powder spectra mimicking isotropic solution-phase spectra. Lorentzian line shape functions were applied to a spectrum of single hyperfine ESR transition with an appropriate line width $\Delta B$. Summing up these subspectra gave a whole spectrum in solution.

[59] M. Takahashi, P. Turek, Y. Nakazawa, M. Tamura, K. Nozawa, D. Shiomi, M. Ishikawa, M. Kinoshita, Phys. Rev. Lett. 1991, 67, 746-748.

[60] M. Nishizawa, D. Shiomi, K. Sato, T. Takui, K. Itoh, H. Sawa, R. Kato, H. Sakurai, A. Izuoka, T. Sugawara, J. Phys. Chem. B, 2000, 104, 503509.

[61] K. Yamaguchi, Chem. Phys. Lett. 1975, 33, 330-335.

[62] M. E. Ali, S. N. Datta, J. Phys. Chem. A 2006, 110, 2776-2784

[63] Y. Kanzaki, D. Shiomi, T. Ise, K. Sato, T. Takui, Polyhedron 2007, 26, 1890-1894.

[64] M. Capdevila-Cortada, J. Ribas-Arino, J. J. Novoa, J. Chem. Theory Comput. 2014, 10, 650-658.

[65] C. D. Sherrill, M. S. Lee, M. Head-Gordon, Chem. Phys. Lett. 1999 302, 425-430.

[66] D. Doehnert, J. Koutecky, J. Am. Chem. Soc. 1980, 102, 1789-1796.

[67] S. Hiraoka, T. Okamoto, M. Kozaki, D. Shiomi, K. Sato, T. Takui, K. Okada, J. Am. Chem. Soc. 2004, 126, 58-59.

[68] D. Shiomi, M. Tamura, H. Sawa, R. Kato, M. Kinoshita, J. Phys. Soc Jpn. 1993, 62, 289-300.

[69] N. M. Gallagher, J. J. Bauer, M. Pink, S. Rajca, A. Rajca, J. Am. Chem. Soc. 2016, 138, 9377-9380.

[70] K. Goto, T. Kubo, K. Yamamoto, K. Nakasuji, K. Sato, D. Shiomi, T. Takui, M. Kubota, T. Kobayashi, K. Yakusi, et al., J. Am. Chem. Soc. 1999, 121, 1619-1620.

[71] M. Tateno, M. Takase, T. Nishinaga, Chem. Mater. 2014, 26, 3804-3810.

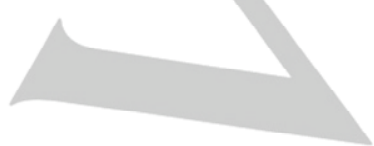




\section{Entry for the Table of Contents}

Radical cation $\pi$-dimers of oligothiophene and co-oligomer with pyrrole unit were demonstrated for the first time to behave as efficient molecular wires for electronic communication between nitronyl nitroxide radicals.

\section{FULL PAPER}
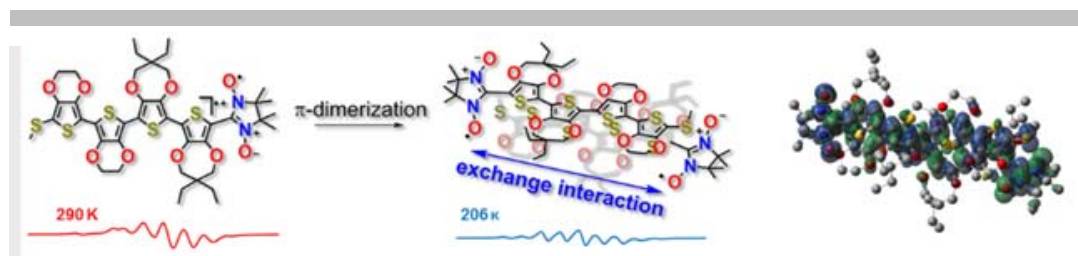

Author(s), Corresponding Author(s)*

Page No. - Page No.

Title 